\title{
On the Dynamics of Gender Differences in Preferences*
}

\author{
Thomas Baudin ${ }^{\dagger}$ and Victor Hiller ${ }^{\ddagger}$
}

May 6, 2015

\begin{abstract}
Investigating gender differences in preferences is crucial to understand gender inequalities and their dynamics. We propose a cultural transmission model in which parents may socialise their sons and daughters differently and we derive gender specific dynamics of preferences. We show how interactions between socialisation behaviours and household's economic decisions shape these dynamics and the long-run gender gap in preferences. We apply our theory to the relative disutility associated with market work vs. housework. Initial and temporary differences in home production technologies can translate into irreversible differences in terms of gender inequalities as such inequalities are magnified by culture.
\end{abstract}

\section{Keywords: Preferences Dynamics, Gender Gap in Preferences, Gender Inequalities, Household Behaviours}

JEL Classification: D13, J16, Z10.

*We would like to thank Matteo Cervellati, David de la Croix and the participants of Overlapping Generations Days at Vielsalm, Conference on the Economics of the Family in honor of Gary Becker at Paris, and seminar participants at the University Paris 1. All remaining errors are ours.

${ }^{\dagger}$ Centre de recherche en démographie et sociétés, UCLouvain. E-mail: thomas.baudin@uclouvain.be

${ }^{\ddagger}$ Corresponding author. Université Paris Est Créteil - ERUDITE \& LEMMA. E-mail: victor.hiller@u-pec.fr 


\section{Introduction}

The last decades of the twentieth century were characterised by a reduction in many aspects of gender inequalities in developed countries. During this period, the pay gap narrowed as well as the gender gap in labour force participation, women caught up with men in terms of college attendance and graduation rates. Even the concept of "gendered occupations" lost part of its meaning (see Goldin 2006). Nevertheless, despite these evolutions and the apparent political voluntarism, gender equality is not a reality yet and some inequalities between men and women seem to persist over time. Women remain under-represented in the highest paid occupations (see Bertrand and Hallock 2001 or Wolfers 2006) and their life cycle earnings after having completed an MBA are significantly lower than those of men with a similar educational attainment (see Bertrand et al. 2010). Gemici and Wiswall (2014) also underline that the drop in the gender gap in college graduation hides important inequalities, with women significantly less likely than men to major in business or science.

A large literature investigates the roots of changes and permanence in gender inequality. A growing number of papers in experimental economics stress the role played by gender differences in preferences. They mainly conclude that women adopt less risky and less competitive behaviours than men (see Croson and Gneezy 2009 for an extensive survey) and hypothesise that these differences may partly explain the persistence of gender inequality. A recent article by Buser et al. (2014) shows that differences in preferences indeed matter. They consider secondary school students in the Netherlands and show that although boys and girls display a similar level of academic ability, boys are more likely to choose studies that are more science intensive. Then, they show that the gender gap in competitiveness (as measured in the lab) accounts for a substantial part of this difference. Zafar (2012) also find evidences on the central role played by gender differences in preferences to explain the gender gap in choice of college major among students at Northwestern University. In particular, they show that these choices are partly driven by the fact that males care more about the pecuniary outcomes in the workplace while females on the non-pecuniary outcomes as the possibility to conciliate work and family life. Bosquet et al. (2013), in a study focusing on academic promotion in France, also find evidence that gender differences in competitiveness partly explain why female professors remain under-represented in French universities. Finally, Flory et al (2014) use field experiment to show that the stronger male's inclination toward competition and their lower aversion to uncertainty compared to women shapes job entries and then the gendered composition of labor markets. This body of findings makes it crucial to analyse the evolution of the gender gap in preferences. Our paper contributes to this research program.

The debate about the origins of gender differences in preferences focuses on the distinction between nature and nurture. On the nature side, Batsesup et al. (2002), for instance, highlight the role of testosterone in competitiveness. On the other side, a large set of studies provide evidence that nurture and thus culture play a role. Following the pioneering work of anthropologists like 
Mead (1935), ${ }^{1}$ Gneezy et al. (2009) study the roots of gender differences in competitiveness within two distinct cultures: the patriarchal society of the Massai in Tanzania and the matrilineal society of the Khasi in India. The authors show that, in the patriarchal Massai society, men compete more than women; but the result is reversed among the Khasi. ${ }^{2}$ These findings suggest that gender differences in preferences are not only due to biological or evolutionary reasons, but also to culture. Even more striking evidence of the role of nurture is provided by Andersen et al. (2013). They study gender differences in the willingness to compete of children aged 7 to 15 in both matrilineal (Khasi) and patriarchal (Kharbi) villages in Northeast India. They show that, in both societies, there are no gender differences at the age of 7 . However, in the patriarchal society, 15-year-old boys are significantly more likely to compete than 7-year-old boys, and the reverse is true for girls. Conversely, in matrilineal society the average behaviour of children does not change. These results emphasise the role played by the socialisation process in the emergence of gender differences in preferences.

Based on these findings, our article constitutes, as far as we know, a first attempt to provide a theoretical framework to model the cultural emergence and evolution of gender differences in preferences. Our framework is built on the cultural transmission model proposed by Bisin and Verdier (2001). They consider that parents may exert some costly efforts in order to transmit one particular preference to their children. Since parents are altruistic (in a paternalistic way), the intensity of this effort depends on the utility that they expect for their children when holding this preference. A large number of papers explore the transmission of various cultural traits through this model (see Bisin and Verdier 2011 for a survey). However, in most of these applications, parents choose the same socialisation effort for all the children within the family. Indeed, it is assumed that the expected utility that parents associate with one particular trait is the same for all children. We depart from Bisin and Verdier's model both (i) by considering a perfect altruism from parents to children $^{3}$ and (ii) by allowing expected utility of children to differ for boys and girls. As a result, parents may choose to socialise their sons and daughters differently. We are then able to characterise the joint dynamics of preferences within both populations of males and females. We show that these dynamics are merely driven by the nature of complementarities within the household. Hence, once the type of complementarity is identified, we can predict the future evolutions of gender differences in preferences.

1. From 1935 and based on her observations of three New Guinea peoples, Mead states that cultural factors are more important than biological ones in shaping male and female behaviours.

2. In a similar spirit, Gong et al. (2014) compare gender differences in social preferences between two Chinese ethnic groups: the matrilineal Mosuo and the Patriarchal Yi. In a dictator experiment, they find that men are more prone to share their payoffs than women within the Mosuo society, while they find no gender differences in Yi society. Gong and Yang (2012) find that women are more risk-averse than men in both societies, but that the gender gap is smaller in the Mosuo.

3. Some cultural transmission papers also adopt this assumption (see, for instance, Doepke and Zilibotti 2008). Saez-Marti and Zilibotti (2008) review the similarities and differences between perfect altruism and paternalistic altruism approaches. 
In some cases, even if (i) boys and girls are born without ex-ante differences, i.e. nature plays no role; (ii) parents are perfectly altruistic and aim at maximising their children's expected utilities; and (iii) preferences are initially equally distributed between genders, differences in preferences between men and women may emerge and persist over time. This result arises in the case of asymmetric complementarity, i.e. when each spouse is better-off if his/her preferences are the opposite of his/her mate's. This relies on the fact that, when they are adults, each girl (resp. boy) is matched with a man (resp. woman) to form a household. Thus, the gender of their children, even if it is associated with no exogenous differences, constitutes a coordination device for parents. If the latter expect one cultural trait to be prevalent within the population of men (resp. women) for the next generation, they have an incentive to transmit a different cultural trait to their daughters (resp. sons). Finally, expectations may be self-fulfilling and gender differences in preferences emerge.

Obviously, the type of complementarity that prevails depends on technology, markets and institutions, as well as on more deeply rooted gender differences. In this sense, we do not deny the role played by nature, since the socialisation choices made by parents may reinforce biological differences. Then, our framework constitutes a powerful tool to analyse the impact of economic and socio-institutional shocks on the long-run distribution of preferences across genders. To illustrate these phenomena, we applied our general model to specific cultural traits.

In our baseline application, we consider that both members of a household collectively decide the time that each spouse has to spend on market activities and home production. Moreover, individuals may exhibit "family-oriented" or "market-oriented" preferences and the type of preference determines the relative disutility of each activity. In this framework, we show that for a low household productivity, the economy converges towards an equilibrium in which men and women display distinct preferences. However, a technological improvement may destabilise the inegalitarian equilibrium and induce the progressive convergence towards an equilibrium in which both men and women are "market-oriented". Along the convergence path, the gender gap in occupation is progressively filled.

We also emphasise the consequences of an inegalitarian bargaining power on the long-run distribution of preferences. We show that, everything being equal, a patriarchal society, where men have a higher bargaining power than women, is more likely to reach an equilibrium in which women are "family-oriented" and men are "market-oriented" than a society where bargaining powers are equal. This last result echoes the previously cited experimental findings (Gneezy et al 2009, Gong et al. 2014, Andersen et al 2012, and Gong and Yang 2012). It also implies that a given technological shock would have different consequences on the dynamics of preferences in two societies which differ in their level of patriarchy.

Then, we enrich our baseline application by considering that the intra-household bargaining power may depend on the average labour supply behaviour of males and females. In this case, the distribution of preferences, by shaping these behaviours, determines the relative bargaining power 
of men and women. Hence, the nature of the complementarity within the household becomes endogenous as it depends on the distribution of preferences. It implies that long-run steady states characterised by persistent gender differences in preferences may coexist with a stable equilibrium in which men and women exhibit similar preferences. Then, two countries initially characterized by distinct technologies may end up with a quite different gender gap in preferences even if their technological discrepancies caught up afterwards. This result may be backed up by Alesina et al. (2013) who show that countries characterized by agricultural forms that rely heavily on physical strength at low development stages are currently more likely to exhibit inegalitarian gender stereotypes.

This article relates to research about the intergenerational transmission of preferences initiated by Bisin and Verdier (2001). Escriche (2007) and Escriche et al (2004) apply this framework to explain the evolution of family-related preferences among women and how this evolution may interact with labour market discrimination and the training policies of firms. Our application builds on these two papers. Indeed, we are interested in the dynamics of the same kind of preference. However, we endogenise the distribution of preferences within both the populations of males and females, while Escriche (2007) and Escriche et al (2004) consider men's preferences to be exogenous. ${ }^{4}$ We also enrich their approach by offering a central role to interactions between spouses within the household, a dimension that is absent from their work.

More broadly, we propose a framework in which the distribution of preferences evolves jointly within two populations. To our knowledge, it is the first attempt to take into account the fact that the evolution of traits within one population may also depend on the distribution of preferences within another population. Once again, we are convinced that gender differences in preferences is a relevant issue to explore this kind of phenomenon since men and women will doubtless interact at the adult age within a household. Finally, our paper also contributes to the recent literature on symmetry breaking (see, for instance, Matsuyama 2002). Indeed, as mentioned previously, we show that gender differences in preferences may endogenously emerge from a fully symmetric environment.

The rest of the paper is organized as follows. Section 2 exposes the model and Section 3 its dynamics properties. In these two sections, we keep a general framework without specifying particular preferences. Our application is proposed in Section 4. Section 5 concludes.

\section{The Model}

We consider an economy populated by overlapping generations of men and women. At each date $t$, the populations of males $(m)$ and females $(f)$ are equally sized. Each individual lives for two periods: childhood and adulthood. Preferences may be of two types $\{a, b\}$ and are acquired during childhood. The proportion of adults with preferences $a$ at date $t$ is denoted $q_{t}^{s}$. During adulthood,

4. In a different framework, Fernández et al (2004) propose a model in which men's disutility from a working wife is endogenous, while the preferences of women are exogenous and stable. 
each male is randomly matched with a female to form a household. Finally, each household has two children, one boy and one girl, and parents spend efforts in order to shape their children's preferences. For simplicity, we assume the matching to be random and make divorce impossible.

\subsection{Preferences Acquisition}

Preferences acquisition by children depends on the joint action of the family and society. In line with Bisin and Verdier (2001), we assume that parents may make an effort to transmit a specific preference to each of their children, the effort that is provided and the preference that is transmitted are not necessarily the same for both children. For simplicity, the probability that an offspring of sex $s$ directly adopts the preference chosen by his/her parent at time $t$ equals the socialisation effort which is denoted $\tau_{t}^{s, i}$. If this direct transmission fails, the child adopts the cultural trait of a role model randomly picked within the population of adults of the same gender. ${ }^{5}$ Hence, we derive the probability $P_{t}^{s, i}$ that a child of gender $s$ and born at $t$ adopts preference $i$ :

$$
\begin{gathered}
P_{t}^{s, a}=\tau_{t}^{s, a}+\left(1-\tau_{t}^{s, a}-\tau_{t}^{s, b}\right) q_{t}^{s} \\
P_{t}^{s, b}=\tau_{t}^{s, b}+\left(1-\tau_{t}^{s, a}-\tau_{t}^{s, b}\right)\left(1-q_{t}^{s}\right)
\end{gathered}
$$

As will become clear later, $\tau_{t}^{s, a}$ and $\tau_{t}^{s, b}$ cannot be simultaneously positive such that the probabilities $P_{t}^{s, a}$ and $P_{t}^{s, b}$ belong to $[0,1]$.

\subsection{Household Decisions}

Households make two kinds of decisions: socialisation and economic decisions. Socialisation decisions consist in the choice of $\tau_{t}^{s, i} \in[0,1]$ for all $i \in\{a, b\}$ and $s \in\{f, m\}$. Since parents are altruistic, these choices are made in order to maximise the expected utility of their children, subject to the fact that the efforts to transmit a culture are costly. Let us denote by $C\left(\tau_{t}^{s, i}\right)$ the utility cost of an effort $\tau_{t}^{s, i}$. Economic decisions are captured abstractly: a spouse $s$, characterised by preferences $i \in\{a, b\}$ and living with a partner of preference $j \in\{a, b\}$, chooses $x^{s, i}(j) \in X$ and derives a utility $v^{i}\left(x^{s, i}(j)\right)$ from this choice. The vector $x^{s, i}(j)$ contains any kind of economic decisions which are not related to socialization like consumption or leisure. Then, the expected utility of an adult $s$ with preference $i$ and living with a type $j$ spouse at date $t$ is written:

$$
V_{t}^{s, i}(j)=v^{i}\left(x^{s, i}(j)\right)+\sum_{\sigma \in\{m, f\}} \sum_{l \in\{a, b\}}\left[P_{t}^{\sigma, l} \hat{V}_{t+1}^{\sigma, l}-C\left(\tau_{t}^{\sigma, l}\right)\right]
$$

5. Girls are influenced only by female role models, while boys are influenced only by male role models. This same-gender role model assumption greatly simplifies the analysis of the dynamics. Several studies in sociology and psychology find that females are indeed much more likely to choose female role models, while the reverse is true for males (see, for instance, Gilbert et al. 1983, Wohlford et al. 2006, or Bricheno and Thornton 2007). 
with $\hat{V}_{t+1}^{\sigma, l}$ the expected utility of an adult of gender $\sigma \in\{m, f\}$ holding preferences $l \in\{i, j\}$ at date $t+1$. The first term of expression (3) is the utility derived from economic decisions, while the second term corresponds to the expected utility of children, net of socialisation costs. Notice that, with this formulation, the way people evaluate their children's future well-being is not subject to gender differences.

Within the household, decisions are made collectively in a cooperative way. The two spouses in a couple $(i, j),{ }^{6}$ choose $\tau_{t}=\left(\tau_{t}^{f, a}, \tau_{t}^{f, b}, \tau_{t}^{m, a}, \tau_{t}^{m, b}\right) \in[0,1]^{4}$ and $x=\left(x^{s, i}(j), x^{-s, j}(i)\right) \in X^{2}$ in order to maximise the following household utility function:

$$
W_{t}^{i, j}\left(x, \tau_{t}\right)=\mu^{s} V_{t}^{s, i}(j)+\left(1-\mu^{s}\right) V_{t}^{-s, j}(i)
$$

subject to technological and budget constraints. These constraints are summarised by a choice set denoted by $Y \subseteq X$. The variable $\mu^{s}$ captures the balance of power within the household, when $\mu^{s}$ increases, the bargaining power of spouse $s$ increases.

As usual in the literature, economic and socialisation decisions can be analysed separately. Indeed, they enter as separable arguments in the household utility and the costs of socialisation are expressed in terms of utility and then do not enter the household budget constraint. Let us first consider economic decisions and define:

$$
\left(\tilde{x}^{s, i}(j), \tilde{x}^{-s, j}(i)\right) \equiv \arg \max _{x \in Y^{2}} \mu^{s} v^{i}\left(x^{s, i}(j)\right)+\left(1-\mu^{s}\right) v^{j}\left(x^{-s, j}(i)\right)
$$

and

$$
u^{s, i}(j)=v^{s, i}\left(\tilde{x}^{s, i}(j)\right)
$$

$u^{s, i}(j)$ is the indirect utility that an adult $s \in\{f, m\}$ with preferences $i \in\{a, b\}$ derives from sharing his/her life with an adult of type $j \in\{a, b\}$. Note that $u^{m, i}(j)$ and $u^{f, i}(j)$ are exogenous ${ }^{7}$ and that they can differ. These differences may come either from biological discrepancies between males and females or from the unequal treatment of men and women within the household or within society. ${ }^{8}$ As a direct implication, even if males and females have the same preferences, they do not necessarily get the same indirect utility.

We can now solve the socialisation problem. In order to obtain close form solutions, we assume that socialisation costs are $C\left(\tau_{t}^{s, i}\right)=\left(\tau_{t}^{s, i}\right)^{2} / 2 k$, where $k$ is a parameter measuring the efficiency of

6. In our notations, within a couple $(i, j)$, the spouse of gender $s$ displays preferences $i$, while the spouse of gender $-s$ displays preferences $j$.

7. Indirect utilities could depend on $q_{t}^{s}$ in many ways. In sub-section 4.3 , we provide a simple example where negotiation powers of spouses are shaped by average behaviours of each gender on the labor market.

8. For instance, the existence of a gender wage gap will translate into different labour supply behaviours between men and women, such that $u^{f, i}(j) \neq u^{m, i}(j)$. Differences between $u^{m, i}(j)$ and $u^{f, i}(j)$ may also come from an unequal distribution of the bargaining power within the household $\left(\mu^{s} \neq 1 / 2\right)$. 
the socialisation technology. Economic choices being made, a household $(i, j)$ chooses $\tau_{t}$ in order to maximise:

$$
\mu^{s} u^{s, i}(j)+\left(1-\mu^{s}\right) u^{-s, j}(i)+\sum_{\sigma \in\{m, f\}} \sum_{l \in\{a, b\}}\left[P_{t}^{\sigma, l} \hat{V}_{t+1}^{\sigma, l}-\left(\tau_{t}^{\sigma, l}\right)^{2} / 2 k\right]
$$

Then, plugging expressions (1) and (2) into (7) and solving the maximisation problem, we immediately get that:

$$
\tau_{t}^{s, a}=\max \left\{0, k\left(1-q_{t}^{s}\right)\left[\hat{V}_{t+1}^{s, a}-\hat{V}_{t+1}^{s, b}\right]\right\} \quad \text { and } \quad \tau_{t}^{s, b}=\max \left\{0, k q_{t}^{s}\left[\hat{V}_{t+1}^{s, b}-\hat{V}_{t+1}^{s, a}\right]\right\}
$$

$\tau_{t}^{s, a}$ and $\tau_{t}^{s, b}$ cannot be simultaneously positive because, in that case, $P_{t}^{s, a}$ and $P_{t}^{s, b}$ would not be reached at a minimal cost. However, parents can transmit alternative traits to each of their children, meaning for instance that $\tau_{t}^{f, a}>0$ is compatible with $\tau_{t}^{m, b}>0$. We obtain this result mainly thanks to our assumption of perfect altruism.

The value of the expected utility of children $\hat{V}_{t+1}^{s, i}$ remains to be determined. Since parents are perfectly altruistic, we must have:

$$
\begin{aligned}
& \hat{V}_{t+1}^{s, a}=\hat{q}_{t+1}^{-s} u^{s, a}(a)+\left(1-\hat{q}_{t+1}^{-s}\right) u^{s, a}(b)+\hat{\Sigma}_{t+2} \\
& \hat{V}_{t+1}^{s, b}=\hat{q}_{t+1}^{-s} u^{s, b}(a)+\left(1-\hat{q}_{t+1}^{-s}\right) u^{s, b}(b)+\hat{\Sigma}_{t+2}
\end{aligned}
$$

with $\hat{\Sigma}_{t+2}$ the expected utility that a child will derive from having children herself/himself ${ }^{9}$ and $\hat{q}_{t+1}^{s}$ the expectation of the proportion of $a$-individuals within population $s$ at date $t+1$.

Substituting the expressions of $\hat{V}_{t+1}^{s, i}$ in (8) and defining $A^{s} \equiv u^{s, a}(a)-u^{s, b}(a)$ and $B^{s} \equiv u^{s, b}(b)-$ $u^{s, a}(b)$, we obtain: ${ }^{10}$

$$
\begin{aligned}
\tau_{t}^{s, a} & =\max \left\{0, k\left(1-q_{t}^{s}\right)\left[\left(B^{s}+A^{s}\right) \hat{q}_{t+1}^{-s}-B^{s}\right]\right\} \\
\tau_{t}^{s, b} & =\max \left\{0, k q_{t}^{s}\left[B^{s}-\left(B^{s}+A^{s}\right) \hat{q}_{t+1}^{-s}\right]\right\}
\end{aligned}
$$

The following assumption ensures that $\tau^{s, i} \in[0,1)$ for all $q_{t}^{-s}$ and $\hat{q}_{t+1}^{-s}$.

9. Let us define this expected utility as $\hat{\Sigma}_{t+2} \equiv \sum_{\sigma \in\{m, f\}} \sum_{l \in\{a, b\}}\left[P_{t+1}^{\sigma, l} \hat{V}_{t+2}^{\sigma, l}-C\left(\tau_{t+1}^{\sigma, l}\right)\right]$ which is a function of $\tau_{t+1}$ the optimal socialisation decisions anticipated for the period $t+1$. Note that, at the aggregate level, the socialisation choices made by adults at date $t$ affect $q_{t+1}$ and then impact $P_{t+1}^{s, i}$ and finally $\hat{\Sigma}_{t+2}$. However, this effect is a pure externality for each individual parent. This is the reason why in the end $\hat{\Sigma}_{t+2}$ is independent from parents' socialization decisions.

10. The signs of $A^{s}$ and $B^{s}$ can vary between genders but not within as gender differences in preferences is the only source of heterogeneity within each sex. This is mainly due to the absence of economic inequalities within genders that we assume for simplicity. Relaxing this assumption would greatly complexity our analysis while not altering our result that gender differences in preferences could emerge in situations where no intrinsic difference exists between male and female. 


\section{Assumption 1}

$$
k<\min \left\{\left|\frac{1}{A^{f}}\right|,\left|\frac{1}{B^{f}}\right|,\left|\frac{1}{A^{m}}\right|,\left|\frac{1}{B^{m}}\right|\right\}
$$

Expressions (11) and (12) highlight the crucial role played by $A^{s}$ and $B^{s}$ in parents' socialisation behaviours. Parameter $A^{s}$ (resp. $B^{s}$ ) measures the utility gain or loss for an individual $s$ matched with a person of type $a$ (resp. $b$ ) from having preference $a$ (resp. $b$ ). In other words, it measures the gain an individual derives from having the same preferences as the person he or she is matched with. As developed in the next section, the long-run evolution of preferences will ultimately depend on the sign of $A^{s}$ and $B^{s}$.

Definition 1 We say that:

$i$ Preference a dominates preference $b$ within population $s$ if: $A^{s}>0$ and $B^{s}<0$.

ii Preference $b$ dominates preference a within population $s$ if: $A^{s}<0$ and $B^{s}>0$.

iii Within population $s$, there exists symmetric complementarity between preferences if: $A^{s}>0$ and $B^{s}>0$.

iv Within population $s$, there exists asymmetric complementarity between preferences if: $A^{s}<0$ and $B^{s}<0$.

In the case of symmetric complementarity among women, whatever the preferences of her partner, a female will always be better off if she holds the same preferences. Conversely, if women's preferences display asymmetric complementarity, a wife will be better off if her preferences are the opposite of her husband's. Note that preferences do not necessarily display the same kind of complementarity among men on the one hand and women on the other.

\subsection{The Dynamics}

From the law of large numbers, the proportion of type $a$ individuals within population $s$ for date $t+1\left(q_{t+1}^{s}\right)$, exactly equals the probability $P_{t}^{s, a}$ defined in (1). Then we can rewrite the latter as:

$$
q_{t+1}^{s}=q_{t}^{s}+\left(1-q_{t}^{s}\right) \tau_{t}^{s, a}-q_{t}^{s} \tau_{t}^{s, b}
$$

We obtain an expression of $q_{t+1}^{s}$ as a function of both $q_{t}^{s}$ and $\hat{q}_{t+1}^{-s}$ by plugging the optimal socialisation efforts (11) and (12) into the law of motion of $q_{t}(13)$ :

$$
q_{t+1}^{s}=\left\{\begin{array}{lll}
q_{t}^{s}+\left(1-q_{t}^{s}\right)^{2} k\left[\left(B^{s}+A^{s}\right) \hat{q}_{t+1}^{-s}-B^{s}\right] & \text { if } \quad\left(B^{s}+A^{s}\right) \hat{q}_{t+1}^{-s} \geq B^{s} \\
q_{t}^{s}+\left(q_{t}^{s}\right)^{2} k\left[\left(B^{s}+A^{s}\right) \hat{q}_{t+1}^{-s}-B^{s}\right] & \text { if } \quad\left(B^{s}+A^{s}\right) \hat{q}_{t+1}^{-s}<B^{s}
\end{array}\right.
$$


The dynamics of $q_{t}^{s}$ admits two parts. When $\left(B^{s}+A^{s}\right) \hat{q}_{t+1}^{-s} \geq B^{s}$ is satisfied, all parents transmit preference $a$ to their child $s$, while when this condition is not satisfied, parents transmit the alternative preference. Finally we define:

$$
\bar{q}^{s}=\frac{B^{s}}{B^{s}+A^{s}}
$$

\section{Long-Run Distribution of Preferences}

In this section, we study the long-run distribution of preferences. For ease of presentation, we first assume expectations to be myopic. Later on, we will analyse the case of perfect expectation for a specific configuration of the values of the parameters.

\subsection{Myopic Expectations}

Under myopic expectations, $\hat{q}_{t+1}^{s}=q_{t}^{s}$. The steady state equilibria of the economy are defined as those pairs $\left(\tilde{q}^{m}, \tilde{q}^{f}\right)$ such that $q_{t+1}^{f}=q_{t}^{f}$ and $q_{t+1}^{m}=q_{t}^{m}$. The following Proposition characterises the long-run distribution of cultural traits for each configuration of the parameters.

Proposition 1 The following table lists, for each parameter configuration, the stable steady states (if any). When there is a unique steady state, it is globally stable; when multiple equilibria coexist, they are locally stable; when there is no stable steady state, the economy admits self-sustained oscillations (cycles).

\begin{tabular}{|c|c|c|c|c|c|}
\hline \multicolumn{2}{|c|}{} & \multicolumn{2}{|c|}{$A^{f}>0$} & \multicolumn{2}{c|}{$A^{f}<0$} \\
\cline { 3 - 6 } \multicolumn{2}{|c|}{} & $A^{m}>0$ & $A^{m}<0$ & $A^{m}>0$ & $A^{m}<0$ \\
\hline \multirow{2}{*}{$B^{f}>0$} & $B^{m}>0$ & $(0,0),(1,1)$ & $(0,0)$ & $(0,0)$ & $(0,0)$ \\
\cline { 2 - 6 } & $B^{m}<0$ & $(1,1)$ & cycles & $(1,0)$ & $(1,0)$ \\
\hline \multirow{2}{*}{$B^{f}<0$} & $B^{m}>0$ & $(1,1)$ & $(0,1)$ & cycles & $(0,1)$ \\
\cline { 2 - 6 } & $B^{m}<0$ & $(1,1)$ & $(0,1)$ & $(1,0)$ & $(0,1),(1,0)$ \\
\hline
\end{tabular}

Proof. See Appendix A

Notice that in the case of multiple equilibria and cycles, $\left(\bar{q}^{m}, \bar{q}^{f}\right)$ is an unstable steady state. ${ }^{11}$ Let us now provide a short discussion for each situation listed in Proposition 1.

Unique globally stable egalitarian equilibrium. This case arises when either the same preference dominates for both genders, or one preference dominates for one gender and there exists

11. One major difference between our framework and this of Bisin and Verdier (2001) is that we assume parental perfect altruism, this directly implies that interior steady states are never stable and sometimes even don't exist. One could argue that the absence of cultural heterogeneity within genders at the steady state is a somehow unrealistic result. Nevertheless, one should not forget that cultural heterogeneity within genders exists in our framework as long as the steady state is not reached, a property that does not contradicts contemporaneous facts. 
symmetric complementarity for the other gender. For instance, when preference $a$ dominates for both genders $\left(A^{f}>0, B^{f}<0, A^{m}>0, B^{m}<0\right)$, parents anticipate that, whatever their gender, their children would be better off when being of type $a$. Then all parents transmit preferences $a$ to their two children, so that, in the long run, all males and females will hold preference $a$. Consider now the case in which preference $b$ dominates within the population of males $\left(A^{m}<0, B^{m}>0\right)$, while preferences are symmetric complements within the population of females $\left(A^{f}>0, B^{f}>0\right)$. All parents will transmit cultural trait $b$ to their sons, so that the proportion $q_{t}^{m}$ decreases over time. This increases the incentives to transmit preferences $b$ to daughters too. At the end, the economy converges toward equilibrium $(0,0)$. Once again, cultural homogeneity between genders is reached in the long run.

Unique globally stable inegalitarian equilibrium. This case arises either when alternative preferences dominate in each population, or when one preference dominates for one gender, while there exists asymmetric complementarity for the other gender. Obviously, when preference $b$ dominates within the population of males, while preference $a$ dominates within the population of females, the long-run distribution of cultural traits is $(0,1)$. In the case in which preference $b$ dominates for women, and men's preferences are characterised by asymmetric complementarity, trait $b$ is transmitted to girls and $q_{t}^{f}$ decreases over time. This drop in $q_{t}^{f}$ increases the expected utility associated with the adoption of preferences $a$ for men and thus the incentive to transmit trait $a$ to boys. In the end, the economy converges towards inegalitarian equilibrium $(1,0)$.

Multiple equilibria. Multiple stable equilibria co-exist when preferences display either asymmetric complementarity or symmetric complementarity for both genders. The dynamics for each of these two cases are illustrated by the phase diagrams in Figure 1. In each configuration, there are three steady state equilibria. Nevertheless $\left(\bar{q}^{m}, \bar{q}^{f}\right)$ is a saddle point (see the proof of Proposition 1). The saddle path that converges towards $\left(\bar{q}^{m}, \bar{q}^{f}\right)$ shares the state space between the basins of attraction of the two locally stable equilibria $((0,1)$ and $(1,0)$ in the case of asymmetric complementarity and $(0,0)$ and $(1,1)$ in the case of symmetric complementarity).

Let us focus on the case of asymmetric complementarity (Figure 1(a)). Due to the existence of multiple equilibria, the long-run situation reached by the economy crucially depends on the initial distribution of preferences. However, whatever the equilibrium reached in the long run, it is characterised by an heterogeneity of preferences between men and women. This result comes directly from asymmetric complementarity. Assume for instance that the economy initially locates very close to the $(1,1)$ equilibrium. As it is highly likely that their son and their daughter will be matched with an $a$-type partner, parents try to transmit them preference $b$. As vertical socialisation is not perfectly efficient, a significant proportion of children will nevertheless be socialised by $a$-type role models and adopt the $a$-trait. This progressive adjustment operates until the dynamical system crosses either the vertical $\bar{q}^{f}$ or the horizontal $\bar{q}^{m}$ line. At this moment, coordination mechanisms 


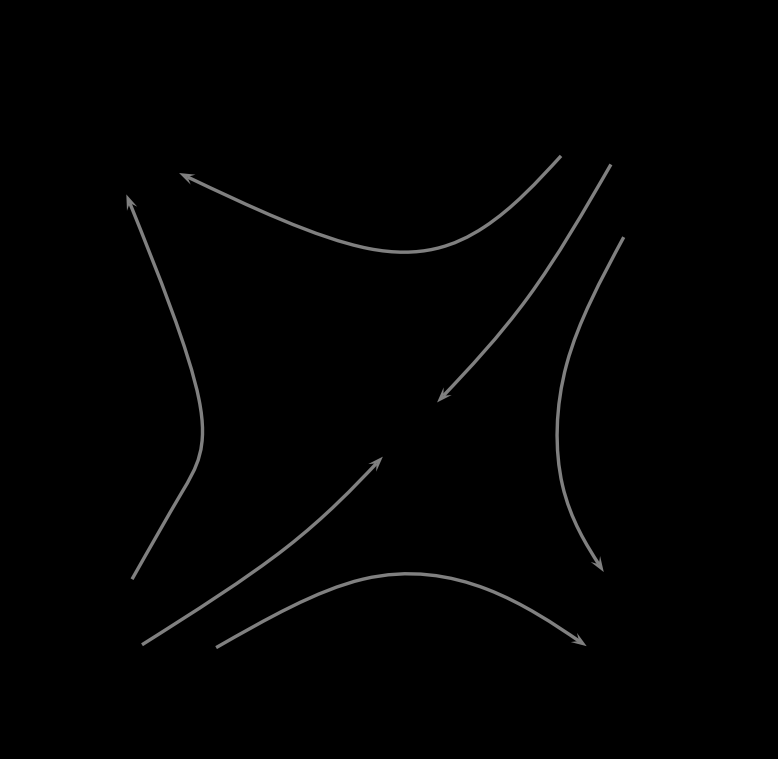

(a) $A^{f}<0, B^{f}<0, A^{m}<0, B^{m}<0$

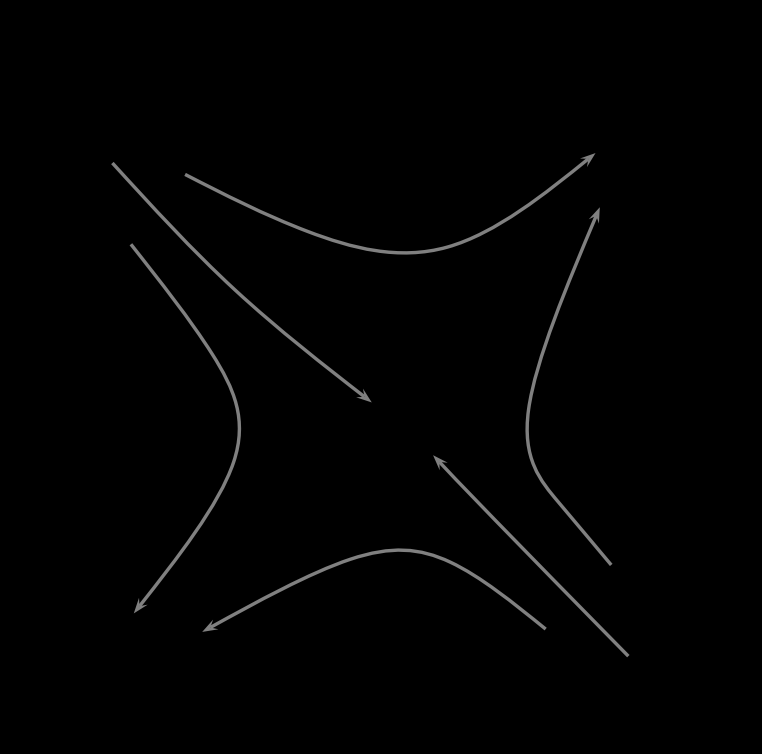

(b) $A^{f}>0, B^{f}>0, A^{m}>0, B^{m}>0$

Figure 1. Multiple equilibria

incite parents to socialise their son and their daughter differently and the economy converges toward an asymmetric equilibrium. An interesting feature of this configuration is that small initial cultural differences between men and women have dramatic effects in the long run. Indeed, if, for instance, the $a$-type trait is more common among men, oblique socialisation will make the dynamics cross the vertical $\bar{q}^{f}$ line instead of the horizontal $\bar{q}^{m}$ one; and the economy converges to the $(0,1)$ equilibrium.

Let us underline that this configuration may hold even if there are no ex-ante differences between genders $\left(A^{f}=A^{m}<0\right.$ and $\left.B^{f}=B^{m}<0\right)$. In this case, whatever the initial distribution of preferences, the economy converges toward one of the two inegalitarian equilibria. Hence, even if nature plays no role and preferences are initially equally distributed between genders, gender inequality may emerge and persist over time. Indeed, in our model, one fundamental difference between genders remains: a woman forms a household with a man, while a man forms a household with a woman. Then, gender may act as a coordination device for parents. This mechanism accounts for the emergence of gender inequalities without ex-ante differences. ${ }^{12}$ This last result may be related to the literature on symmetry-breaking (see, for instance, Matsuyama 2002). Indeed, in the configuration $A^{f}=A^{m}<0$ and $B^{f}=B^{m}<0$, an asymmetric outcome endogenously emerges from a fully symmetric environment.

12. We abstract from modelling same-sex unions and celibacy. Nevertheless, our main results still hold as long as we assume that a positive fraction of individuals are matched with an opposite sex partner. 
Self-sustained oscillations When preferences display asymmetric complementarity for one gender and symmetric complementarity for the other one, the dynamics exhibit one unique steady state $\left(\bar{q}^{m}, \bar{q}^{f}\right)$ which is a spiral source (see the proof of Proposition 1). Hence, the dynamics of the economy exhibit self-sustained oscillations and converge toward a limit cycle as depicted in the following phase diagrams (Figure 2).

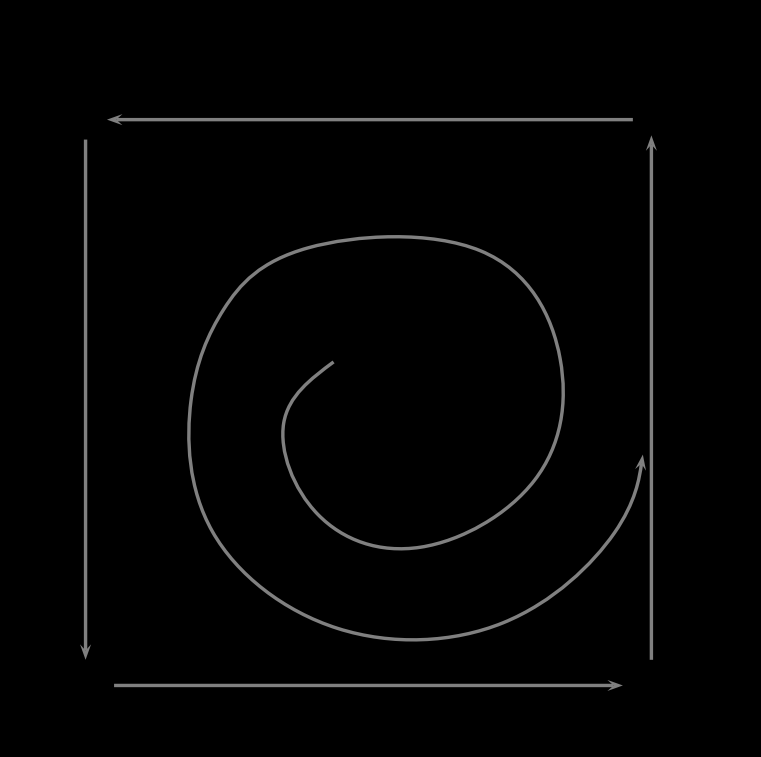

(a) $A^{f}>0, B^{f}>0, A^{m}<0, B^{m}<0$

Figure 2. Cycles

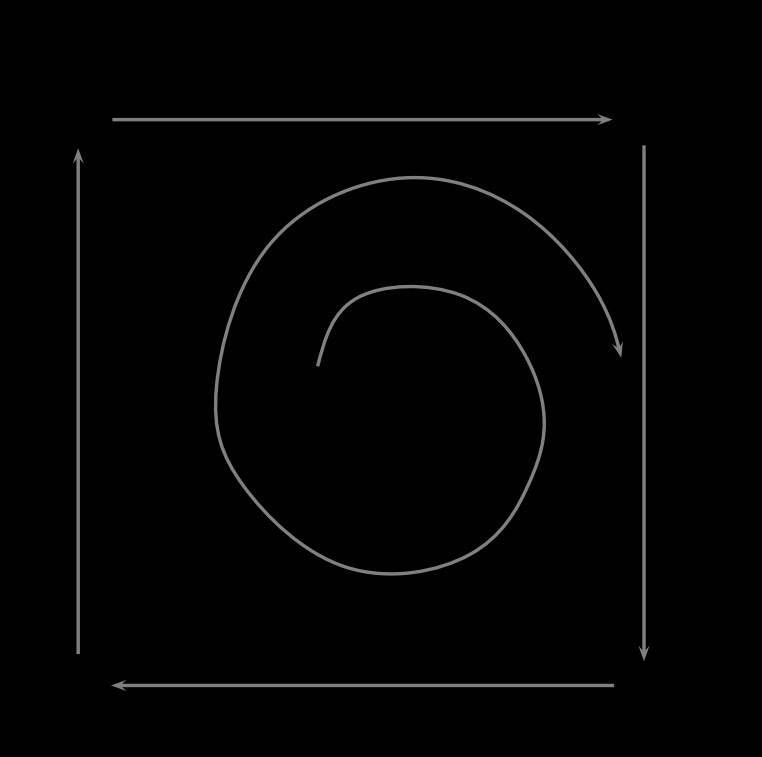

(b) $A^{f}<0, B^{f}<0, A^{m}>0, B^{m}>0$

Let us consider the case depicted in Figure 2(a) (preferences are symmetric complements for females and asymmetric complements for males) and focus on an initial situation in which $q_{0}^{f}<\bar{q}^{f}$ and $q_{0}^{m}<\bar{q}^{m}$. The proportion of both type $a$ males and females is low, it leads parents to transmit type $b$ preferences to their daughters (due to symmetric complementarity) and type $a$ preferences to their sons (due to asymmetric complementarity). Consequently, $q_{t}^{f}$ is reduced while $q_{t}^{m}$ rises and finally overcomes $\bar{q}^{m}$ such that it becomes beneficial to socialise girls in accordance with preferences $a$ and $q_{t}^{f}$ starts to rise. As soon as $q_{t}^{f}$ becomes larger than $\bar{q}^{f}$, parents transmit preferences $b$ to their sons, $q_{t}^{m}$ decreases and so on.

\subsection{Perfect Foresight}

Consider now that expectations are perfect: $\hat{q}_{t+1}^{s}=q_{t+1}^{s}$. In this configuration, all the results obtained in Proposition 1 hold unchanged. In addition, when multiple equilibria co-exist, indeterminacy regions may arise in which expectations are self-fulfilled.

To illustrate this point, we restrict our attention to the case in which there are no ex-ante 
differences between genders $\left(A^{f}=A^{m}=A \text { and } B^{f}=B^{m}=B\right)^{13}$, preferences are characterised by asymmetric complementarity ( $A$ and $B$ are both negative) and $k$ is not too low:

$$
k>\left|\frac{1}{A+B}\right|
$$

Under these assumptions, the dynamical behaviour of the economy is described in Proposition 2.

Proposition 2 Consider that expectations are perfect, both $A$ and $B$ are negative and Assumption 1 and condition (16) hold: the dynamics of the economy exhibit three steady states: $(\bar{q}, \bar{q})$ is a saddle point, and $(1,0)$ and $(0,1)$ are locally stable. Moreover, there is an indeterminacy area I such that for $\left(q_{t}^{m}, q_{t}^{f}\right) \in I$ the expectations that $\left(q_{t+1}^{f}, q_{t+1}^{m}\right)$ will belong to the basin of attraction of $(1,0)$ and the basin of attraction of $(0,1)$ are both self-fulfilling.

Proof. See Appendix B

The results of Proposition 2 may be illustrated by the phase diagram in Figure 3. In this figure, the two hatched areas correspond to the indeterminacy region $I$ while the grey arrows correspond to the saddle path. The basin of attraction of $(1,0)$ (resp. $(0,1))$ is located under (resp. above) this saddle path. ${ }^{14}$

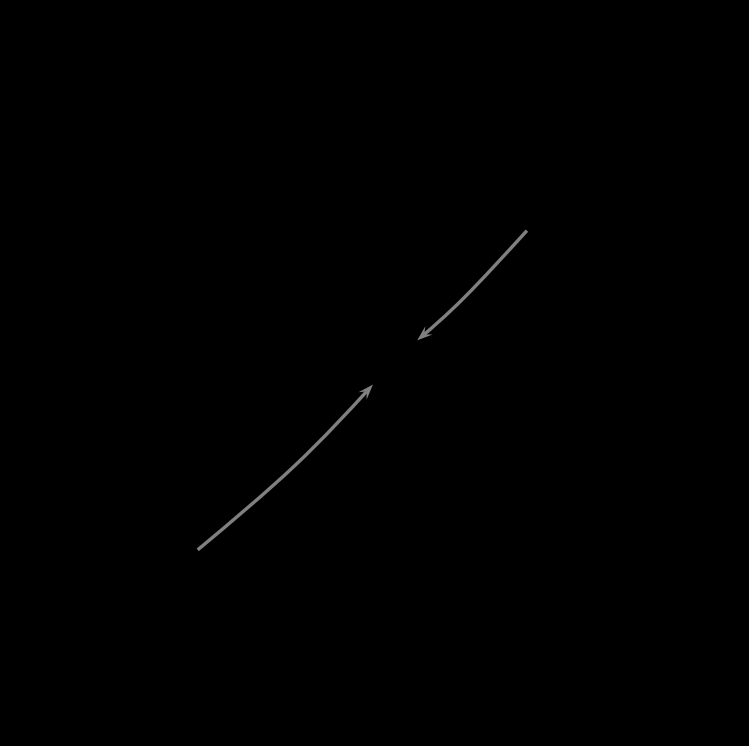

Figure 3. Multiple equilibria and self-fulfilling beliefs

As claimed in Proposition 2, outside the indeterminacy region, the dynamics behave as in the myopic expectation case: multiple steady states exist and the economy converges towards the equilibrium $(0,1)$ (resp. $(1,0)$ ) if it is initially located under (resp. above) the saddle path. Let us now

13. Note that these assumptions imply that $\bar{q}^{f}=\bar{q}^{m}=\bar{q}=B /(A+B)$.

14. See Appendix B for the derivation of Figure 3. 
consider in more detail the case of one economy initially located in the indeterminacy region. In the configuration in which $\left(q_{t}^{m}, q_{t}^{f}\right) \in I$, the distribution of preferences is balanced. Parents have no incentive to perpetuate this egalitarian distribution due to the asymmetric complementarity property. Hence there are two alternatives: either parents aim at transmitting preference $a$ to their daughters and $b$ to their sons or conversely. If all parents coordinate their expectations on one of the two alternatives, these expectations become self-fulfilling. If they believe in particular that other parents will transmit preference $a$ to their daughters and $b$ to their sons, they have an incentive to do the same. At the next date, $q_{t+1}^{f}$ will be higher than $\bar{q}$ and $q_{t+1}^{m}$ will be lower than $\bar{q}$ so that the economy will belong to the basin of attraction of $(0,1)$. For symmetric reasons, if they believe that other parents will transmit preference $a$ to their sons and $b$ to their daughters, the economy will end up in the equilibrium $(1,0)$.

Let us underline that the indeterminacy region $I$ exists only for a sufficiently high value of $k$ (condition (16)). Indeed, for high values of $k$, the marginal cost of socialisation is low, which leads parents to exert a strong transmission effort (see (11) and (12)). Thus, when $k$ increases, the relative weight of vertical vs oblique socialisation rises. This implies a reduction of the backward-looking component of the preferences dynamics, so that one initial condition may be compatible with several self-fulfilling expectations.

Proposition 2 echoes quite interestingly the results of Dolado et al. (2013) who show that selffulfilling expectations and multiple equilibria in pay and housework time allocation may emerge in situations where no ex-ante differences between men and women exist. In their framework, the multiplicity of equilibria comes from statistical discrimination implemented by firms in the provision of paid-for training to workers. In our framework, the multiplicity of equilibria comes from the interactions between men and women within households, such interactions could obviously be shaped by the behaviours of firms on the labor market.

\section{Application}

In the previous section, we showed that socialisation choices, which drive the evolution of preferences, may be separated from economic choices. Thanks to Proposition 1, we can directly deduce the dynamic behaviour of one economy from the analysis of the kind of complementarities which arise within households (signs of $A^{s}$ and $B^{s}$ ). Hence, our model constitutes a very tractable framework that could be easily used to analyse why some differences in preferences persist, while others vanish over times. In Section 4.1, we provide a simple application of our model where individuals can be either "family-oriented" or "market-oriented". The two types of individuals differ according to the relative disutility that they associate to market work vs housework. Comparative statics, which are implemented in Section 4.2, illustrate how technological and institutional factors, by shaping the way spouses interact, may account for the presence of gender differences in preferences. 
We show in particular that, if home production technologies are not efficient enough, asymmetric complementarity arises and gender differences in preferences emerge and perpetuate themselves. However, a positive shock on the efficiency of home production may make the preference for market activities dominant, allowing for a progressive disappearance of gender differences with respect to these specific preferences. We also highlight the role played by the gender gap in bargaining power to understand the type of inegalitarian equilibrium which is reached. For a given technological level, a patriarchal society, i.e. a society in which men have a higher bargaining power, is more likely to converge toward an equilibrium in which men display "market-oriented" preferences and women are "family-oriented" than an economy characterised by a more balanced bargaining power between genders. $^{15}$

The simplicity of our baseline model has obviously some adverse side effects. Within one population, one specific type of complementarity arises such that, for a fixed technological and institutional environment, the long-run distribution of preferences is either egalitarian or inegalitarian. Hence, our basic framework fails to explain why two comparable countries in terms of institutional and technological achievements may be characterised by distinct levels of gender differences in preferences. In a second application (Section 4.3), we show how our basic framework may be enriched to overcome this weakness. Indeed, we show that when bargaining power is endogenous, the type of complementarity within households depends on the current distribution of preferences, implying that egalitarian and inegalitarian stable steady states may co-exist.

\subsection{The Baseline Model}

Let us consider a given household. Each spouse is endowed with one unit of time that is shared between work on the market and housework, $l^{s, i}$ denotes the housework time of the spouse of gender $s$ characterised by preferences $i$. We assume that the couple has to devote an incompressible period of time $\lambda \in(0,1)$ to housework, no matter how the burden is shared. The parameter $\lambda$ corresponds to the minimum amount of time required to maintain the home and care for the family. For ease of simplicity, we assume that this time is constant. It is nevertheless a function of technology as a technological shock (like the spread of new household appliances) can lower it. The time which is not devoted to housework is spent in the workforce, $w$ denotes the market wage by unit of time.

Preferences heterogeneity stems from the relative disutility associated with work on the market. We consider in particular that $a$-type individuals are "job-oriented", i.e. they experience disutility only during housework. Conversely, b-type individuals are "family-oriented", i.e. their disutility

only stems from time they spend on the market. ${ }^{16}$ This assumption is obviously extreme and is

15. Then, in line with experimental studies by Gneezy et al. (2009) or Andersen et al. (2012), we illustrate the fact that the level of patriarchy within a society affects the differences between men and women in terms of preferences. 16. By considering this kind of preference, we are in the line with Escriche (2007) and Escriche et al. (2004). However, while these two papers assume that men are biologically "job-oriented" and only analyse the evolution of the proportion of "family-oriented" women, we endogenise the distribution of preferences within both populations. 
made in order to keep the analysis simple. Our results would hold unchanged if we had considered that the relative disutility associated with housework is lower for $a$-type individuals than for $b$-type ones. ${ }^{17}$ Formally, the preferences of an individual of gender $s$ with preferences $i$ are:

$$
v^{i}\left(c^{s, i}, l^{s, i}\right)=\ln c^{s, i}-F_{i}\left(l^{s, i}\right)
$$

with

$$
F_{i}\left(l_{i}^{s}\right)=\left\{\begin{array}{llc}
\left(l^{s, i}\right)^{2} & \text { if } & i=a \\
\left(1-l^{s, i}\right)^{2} & \text { if } & i=b
\end{array}\right.
$$

We consider that males and females earn the same wage on the market and have the same productivity at home. However, in order to account for the influence of patriarchal norms, we consider that men and women may have a different bargaining power. A couple composed of a male of type $i$ and a female of type $j$ aim at maximising the following collective household utility function with respect to $\left\{c^{m, i}, c^{f, j}, l^{m, i}, l^{f, j}\right\}$ :

$$
W_{t}^{i, j}\left(c^{m, i}, c^{f, j}, l^{m, i}, l^{f, j}\right)=\mu v^{i}\left(c^{m, i}, l^{m, i}\right)+(1-\mu) v^{j}\left(c^{f, j}, l^{f, j}\right)
$$

The bargaining power of men is denoted by $\mu \in[0,1]$. As a first step, we consider that $\mu$ is exogenously given. In line with Doepke and Tertilt (2009) or Fernández (2014), we assume that it constitutes a measure of the level of patriarchy of the economy. ${ }^{18}$ The case of an endogenous intra-household bargaining power will be addressed in Section 4.3.

Spouses maximise (19) under the following time and budget constraints:

$$
\begin{gathered}
l^{m, i}+l^{f, j} \geq \lambda \\
c^{m, i}+c^{f, j}=\left(2-l^{m, i}-l^{f, j}\right) w
\end{gathered}
$$

In the following, we will assume that $\lambda>(3-\sqrt{5}) / 2$. This condition ensures that the time constraint (20) is always binding. ${ }^{19}$ Then, the budget constraint $(21)$ translates into $c^{f, i}+c^{m, j}=(2-\lambda) w$.

Let $x^{s, i}(j)=\left(c^{s, i}(j), l^{s, i}(j)\right)$ be the set of economic choices made by an agent of gender $s$ with

17. Here, we consider that differences in preferences are directly associated with the disutility coming from each kind of activity. This can be viewed as a short-cut to a more general model in which differences in preferences would be associated with risk aversion and/or taste for competition. As the labour market is a more competitive and risky environment than the household, we would obtain the same kind of result: $b$-type agents would be more averse to risk and competition and would prefer to stay at home, while the reverse would be true for $a$-type agents.

18. This view is consistent with the differences between matrilineal Khasi and patriarchal Kharbi societies as reported by Andersen et al. (2012): "The Khasi husband dwells in a household in which he has no authority or property [...] The Kharbi society, on the other hand, is organized in the usual patriarchal structure, in that men possess ownership of the land and have power over monetary decisions of the households. "See also Shu et al. (2013) on the relationship between patriarchy and bargaining power within the household.

19. The time constraint is not binding for low values of $\lambda$. Indeed, since $b$-type individuals experience disutility when working on the market, they could optimally choose to stay at home for a period longer than $\lambda$. 
preference $i$ who is matched with an agent of type $j$. Consumption choices are :

$$
c^{m, i}(j)=\mu(2-\lambda) w \quad \text { and } \quad c^{f, i}(j)=(1-\mu)(2-\lambda) w \quad \text { for all } \quad i, j \in\{a, b\}^{2}
$$

These decisions are independent from cultural traits but not from gender; indeed equation 20 is binding what makes the income of the household independent from the distribution of housework time and there is no gender differences in preferences with respect to consumption. Conversely, each spouse's labour supply depends on her/his own preferences and the preferences of her/his mate. Within homogamous $\{a, a\}$ and $\{b, b\}$ couples, the sharing of housework depends on each spouse's relative bargaining power:

$$
\begin{array}{ll}
l^{f, a}(a) & =\mu \lambda \\
l^{f, b}(b) & =\left\{\begin{array}{ll}
\lambda & \text { if } \mu<\hat{\mu}_{1}(\lambda) \\
1-\mu(2-\lambda) & \text { if } \mu \in\left[\hat{\mu}_{1}(\lambda), \hat{\mu}_{2}(\lambda)\right] \\
0 & \text { if } \mu>\hat{\mu}_{2}(\lambda)
\end{array} l^{m, a}(a)=(1-\mu) \lambda\right. \\
& \text { if } \mu<\hat{\mu}_{1}(\lambda)
\end{array}
$$

with

$$
\hat{\mu}_{1}(\lambda) \equiv \frac{1-\lambda}{2-\lambda} \quad \text { and } \quad \hat{\mu}_{2}(\lambda) \equiv \frac{1}{2-\lambda}
$$

In turn, heterogamous couples are always characterised by a specialisation of tasks: $a$-type agents fully specialise in labour market activities as they do not experience any loss coming from this activity, while $b$-type agents carry out all the domestic duties as this activity is not a source of disutility for them. Their remaining time is devoted to labour market activities:

$$
l^{f, a}(b)=l^{m, a}(b)=0 \quad \text { and } \quad l^{f, b}(a)=l^{m, b}(a)=\lambda
$$

By plugging the optimal behaviours (23) and (25) into the utility function (17), we obtain each individual's indirect utility as a function of his/her preferences and the preferences of her/his partner. Then, we deduce the following values for $A^{f}, A^{m}, B^{f}$ and $B^{m}$ :

$$
\begin{array}{ll}
A^{f} & =-\mu^{2} \lambda^{2}+(1-\lambda)^{2} \\
B^{f}=\left\{\begin{array}{lll}
-(1-\lambda)^{2} & \text { if } & \mu<\hat{\mu}_{1}(\lambda) \\
-\mu^{2}(2-\lambda)^{2} & \text { if } & \mu \in\left[\hat{\mu}_{1}(\lambda), \hat{\mu}_{2}(\lambda)\right] \\
-1 & \text { if } & \mu>\hat{\mu}_{2}(\lambda)
\end{array} \quad, \quad B^{m}=\left\{\begin{array}{lll}
-1 & \text { if } & \mu<\hat{\mu}_{1}(\lambda) \\
-\mu^{2}(2-\lambda)^{2} & \text { if } & \mu \in\left[\hat{\mu}_{1}(\lambda), \hat{\mu}_{2}(\lambda)\right] \\
-(1-\lambda)^{2} & \text { if } & \mu>\hat{\mu}_{2}(\lambda)
\end{array}\right.\right.
\end{array}
$$

$B^{f}$ and $B^{m}$ are both negative meaning that, when matched with a $b$-type agent, it is always preferable to have preferences $a$. It comes from the result of full specialisation within heterogamous couples: if an $a$-type agent is matched with a $b$-type one, he will specialise in labour market activities which do not constitute a source of disutility. Conversely, if a $b$-type is matched with another $b$, 
then each of them spends some time on the labour market and experiences the associated disutility. On the other hand, the sign of $A^{f}$ and $A^{m}$, and accordingly the long-run distribution of preferences, depends on parameter values. Starting from the expressions of $A^{f}$ and $A^{m}$ given in (26), we can define:

$$
\tilde{\mu}_{1}(\lambda) \equiv \frac{1-\lambda}{\lambda} \quad \text { and } \quad \tilde{\mu}_{2}(\lambda) \equiv \frac{2 \lambda-1}{\lambda}
$$

such that $A^{f}$ is positive when $\mu<\tilde{\mu}_{1}(\lambda)$ and is negative otherwise and $A^{m}$ is positive when $\mu>\tilde{\mu}_{2}(\lambda)$ and is negative otherwise. Figure 4 depicts the type of complementarity arising within the household according to the values of $\mu$ and $\lambda$.

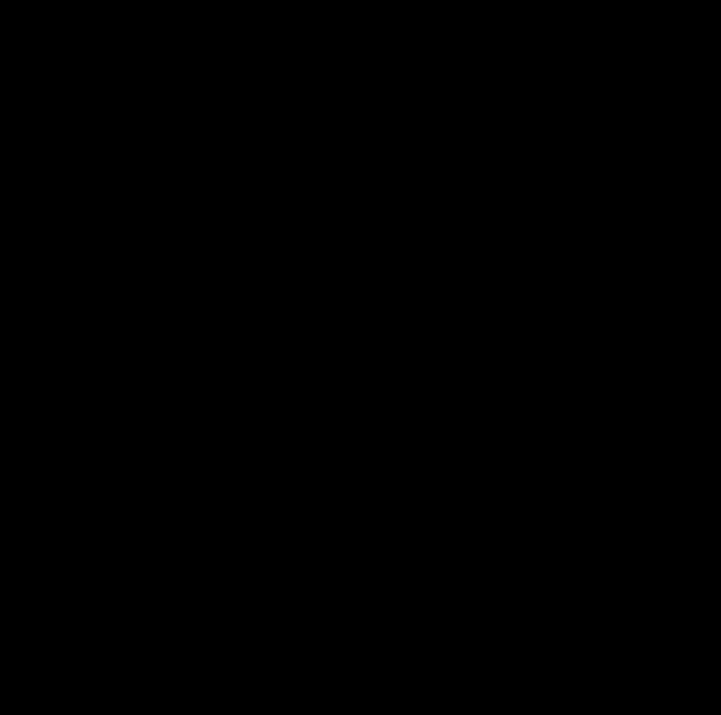

Figure 4. Sign of $A^{f}$ and $A^{m}$ as a function of $\lambda$ and $\mu$

\subsection{Comparative Statics: Technology and Patriarchy}

Let us first consider the impact of $\lambda$ under the assumption of equal bargaining powers, $\mu=1 / 2 .{ }^{20}$ When $\lambda$ is high, $A^{f}$ and $A^{m}$ are negative, while they become both positive if $\lambda$ becomes low enough. Indeed, when an agent is matched with an $a$-type mate, she/he will necessarily have to participate in both housework and the workforce. Then, if the agent is of type $a$, she/he will suffer from the amount of time she/he devotes to housework (increasing in $\lambda$ ). But, if the agent is of type $b$, she/he will suffer from the amount of time spent on the market (decreasing in $\lambda$ ). So if $\lambda>2 / 3$, the first effect overcomes the second one and the agent is better off if she/he is of type $b$. Conversely, if $\lambda<2 / 3$, it is preferable to be an $a$-type agent and share housework time with the partner.

20. In this specific case, there are no ex-ante differences between men and women: $A^{f}=A^{m}$ and $B^{f}=B^{m}$. 
We can now describe the impact of a technological shock increasing the productivity of housework activities. Such a shock corresponds to a fall in $\lambda$. Consider that, initially, the productivity of household appliances is low $(\lambda>2 / 3)$. As depicted in Figure 4, preferences exhibit asymmetric complementarity. Hence, the economy converges toward a situation characterised by a strong differentiation of gender roles: each gender specialises in one kind of occupation (housework or market) and each gender's preferences sustain this separation of tasks. A priori, we do not know which gender is specialised in which task; to comply with historical observation, let us consider that the economy converges towards the $(1,0)$ equilibrium. ${ }^{21}$

Consider now an increase in the productivity of housework activities coming from, for instance, the introduction of new household appliances allowing for a reduction of the time devoted to maintaining the home. Such a shock corresponds to a decrease in $\lambda$. If this decrease is large enough (after the shock, $\lambda<2 / 3$ ), it destabilises the $(1,0)$ equilibrium. Since "job-oriented" preferences dominate, the economy converges towards the $(1,1)$ equilibrium. Hence the proportion of women who prefer to work in a competitive environment increases and the gender gap in housework participation is progressively filled. Obviously, during the transition to the new steady state, the average participation time of males to housework activities increases, while the average participation time of women decreases. Indeed, due to the rise in $q_{t}^{f}$, the proportion of males involved in housework activities increases, as well as the proportion of females who only do half of the housework tasks. ${ }^{22}$

Let us now analyse the impact of $\mu$ on the long-run distribution of preferences. We can see in Figure 4 that, for balanced values of $\lambda$, the economy will converge towards the equilibrium $(1,0)$ (resp. $(0,1))$ when $\mu$ is high (resp. low), while for intermediate values of $\mu$ the unique steady state is $(1,1)$. Hence, the distribution of bargaining powers affects gender differences in preferences in the long run. The level of patriarchy also determines which kind of inegalitarian equilibrium is reached $((1,0)$ or $(0,1))$. When $\mu$ is high, the share of housework activities completed by males within homogamous $\{a, a\}$ couples is low so that, when matched with a type $a$ woman, a man is better off when being $a$ himself $\left(A^{m}>0\right)$. Conversely, within an $\{a, a\}$ couple, the wife would complete most housework activities. Consequently, when matched with a type $a$ man, a woman is better off when being $b\left(A^{f}<0\right)$. Then the proportion of type $a$ men (resp. women) increases (resp. decreases) over time and the economy converges towards the equilibrium $(1,0)$. A symmetric reasoning applies for low values of $\mu$ such that the economy converges towards $(0,1)$.

21. The fact that the economy ends up in the $(1,0)$ rather than the $(0,1)$ equilibrium may come from the fact that, initially, the proportion of $a$ trait is higher within the population of males than within the population of females. It may also be the case that the economy was located in an indeterminacy area and the population coordinates on the expectation that women will be more "family-oriented" than men in the coming generations. Finally, and as will be developed below, a slight difference in bargaining power between genders may induce the convergence towards the $(1,0)$ equilibrium.

22. Bianchi (2011) estimates that, from 1965 to 2008, American mothers divided their housework time by almost two (from 31.9 hours per week to 17.4), while American fathers more than doubled their participation in housework activities (from 4.4 hours per week in 1965 to 9.5 hours in 2008). Although we will not provide any quantitative exercise here, our simple model is able to qualitatively reproduce this pattern. 
Then, everything being equal, a patriarchal society in which men have a higher bargaining power than women, is more likely to reach an equilibrium in which women are "family-oriented" and men "market-oriented" than a society in which bargaining powers are equal. This last result echoes experimental findings (Gneezy et al. 2009, Andersen et al. 2012, Gong and Yang 2012 and Gong et al. 2014) showing that market-oriented preferences, such as a low degree of risk-aversion or a high taste for competition, tend to be more prevalent within the population with the higher bargaining power. Our result also implies that a given technological shock would have different consequences on the dynamics of preferences in two societies which differ in their level of patriarchy. Indeed, for a given fall in $\lambda$, the likelihood to remain trapped in an inegalitarian equilibrium is increasing in the degree of inequality that characterises the distribution of power within the household.

\subsection{Endogenous Bargaining Power}

Up to now, we have identified the relative intra-household bargaining power with the exogenously given degree of patriarchy within the society. Recent studies recognise that this bargaining power may be, at least partly, shaped by endogenous forces, such as the gender gap in labour supply (see, for instance, Basu 2006). In this section, we endogenise $\mu$ in a very simple way and we study the consequences for the long-run evolution of gender differences in preferences.

Let us consider once again our baseline model and fix the value of $\lambda$ according to the following assumption:

Assumption $2 \lambda \in(1 / 2,2 / 3)$

Under Assumption 2, the type of complementarity arising within households crucially depends on the value of $\mu$ (see Figure 4). Moreover, it directly comes from (24) and (27) that Assumption 2 implies:

$$
\hat{\mu}_{2}(\lambda)>\tilde{\mu}_{1}(\lambda)>1 / 2>\tilde{\mu}_{2}(\lambda)>\hat{\mu}_{1}(\lambda)
$$

Following Komura (2013), we assume that the intra-household bargaining power, while exogenous at the family level, is endogenous at the society level. More precisely, we consider that $\mu$ is a function of the differences between the average labour supply behaviours of males and females. To keep the analysis as simple as possible, we assume that it depends on the difference between the number of males and females who spent their entire period of time on the labour market. Let us denote this difference $\delta_{t} \equiv n_{t}^{m}-n_{t}^{f}$, with $n_{t}^{s}$ the number of individuals spending their entire period of time on the labour market within population $s$. The relative bargaining power at date $t, \mu_{t}$, will be related to $\delta_{t}$ through the function $\mu\left(\delta_{t}\right)$ which is strictly increasing on $[-1,1]$ with $\mu(-1)=\underline{\mu}, \mu(1)=\bar{\mu}, \mu(0)=1 / 2$ and $\mu(\delta)=\mu(-\delta)$ for all $\delta \in[0,1]$. Moreover, we make the following assumption.

Assumption $3 \bar{\mu} \in\left(\tilde{\mu}_{1}(\lambda), \hat{\mu}_{2}(\lambda)\right)$ and $\underline{\mu} \in\left(\hat{\mu}_{1}(\lambda), \tilde{\mu}_{2}(\lambda)\right)$ 
The restrictions $\bar{\mu}<\hat{\mu}_{2}(\lambda)$ and $\underline{\mu}>\hat{\mu}_{1}(\lambda)$ avoid a corner solution on $l^{f, b}(b)$ or $l^{m, b}(b)$ (see expression (23)). Assumptions $\bar{\mu}>\tilde{\mu}_{1}(\lambda)$ and $\underline{\mu}<\tilde{\mu}_{2}(\lambda)$ ensure that there exist some values of $\delta_{t}$ such that we have $A^{f}<0$ and $A^{m}>0$ or $A^{f}>0$ and $A^{m}<0$ (see Figure 4). Finally, let us define $\hat{\delta}$ as the threshold value of $\delta_{t}$ such that $\mu(\hat{\delta})=\tilde{\mu}_{1}(\lambda)$ or equivalently $\mu(-\hat{\delta})=\tilde{\mu}_{2}(\lambda)$.

From expressions (23) and (25) and under the Assumptions 3, only individuals of type $a$ matched with a type $b$ spouse will specialise in market activities. Then we have:

$$
n_{t}^{m}=q_{t}^{m}\left(1-q_{t}^{f}\right) \quad \text { and } \quad n_{t}^{f}=q_{t}^{f}\left(1-q_{t}^{m}\right) \quad \text { such that } \quad \delta_{t}=q_{t}^{m}-q_{t}^{f}
$$

From this expression and through the properties of the function $\mu\left(\delta_{t}\right)$, we get that:

- $\mu>\tilde{\mu}_{1}(\lambda)$ if $q_{t}^{f}<q_{t}^{m}-\hat{\delta}$

- $\mu \in\left[\tilde{\mu}_{2}(\lambda), \tilde{\mu}_{1}(\lambda)\right]$ if $q_{t}^{f} \in\left[q_{t}^{m}-\hat{\delta}, q_{t}^{m}+\hat{\delta}\right]$

- $\mu<\tilde{\mu}_{2}(\lambda)$ if $q_{t}^{f}<q_{t}^{m}-\hat{\delta}$

Then the plan $\left(q_{t}^{m}, q_{t}^{f}\right)$ may be partitioned into three regions which differ according to the kind of complementarity which prevails within the household. We deduce from Figure 4 that when $q_{t}^{f}<q_{t}^{m}-\hat{\delta}, A^{f}<0$ and $A^{m}>0$. Indeed, in this configuration, the number of men specialised in market work is much higher than the number of women. As a consequence, the relative bargaining power of males is so high (higher than $\tilde{\mu}_{1}(\lambda)$ ) that preferences becomes asymmetric complements within the population of females. Through similar reasoning we conclude that when $q_{t}^{f}>q_{t}^{m}+\hat{\delta}$, $A^{f}>0$ and $A^{m}<0$ and when $q_{t}^{f} \in\left[q_{t}^{m}-\hat{\delta}, q_{t}^{m}+\hat{\delta}\right]$ both $A^{f}$ and $A^{m}$ are positive. This partition is illustrated in Figure 5(a). The dynamical consequences are summarised by the phase diagram depicted in Figure 5(b). ${ }^{23}$ In this diagram, the curves $\bar{q}^{m}\left(q_{t}^{m}\right)$ and $\bar{q}^{f}\left(q_{t}^{f}\right)$ are respectively the stationary locus of $q_{t}^{m}$ and the stationary locus of $q_{t}^{f}$.

As illustrated in this phase diagram, the three equilibria $(0,1),(1,0)$ and $(1,1)$ are locally stable. The reason behind this result is that: $(i)(1,1)$ belongs to a region where preference $a$ dominates for both genders, $(i i)(0,1)$ belongs to a region where there is asymmetric complementarity for men, while preference $a$ dominates among women, and $(i i i)(1,0)$ belongs to a region where there is asymmetric complementarity for women, while preference $a$ dominates among men.

The multiplicity of equilibria implies that initial conditions are fundamental. Indeed, assume that two countries, $\mathcal{A}$ and $\mathcal{B}$, have the same technology. Assume also that in country $\mathcal{A}$, gender differences in preferences are strong while in country $\mathcal{B}$, the initial distribution of preferences is close within both populations. Then, in country $\mathcal{A}$, the labour supply behaviours of males and females tend to be quite different so that the intra-household bargaining power tends to be unequally 23. See Appendix $\mathrm{C}$ for a formal derivation of this phase diagram. 


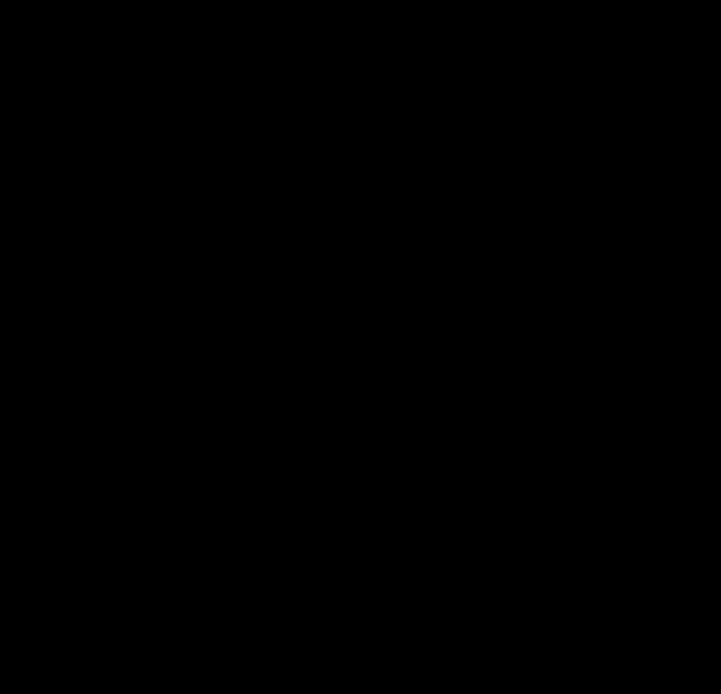

(a) Nature of complementarity

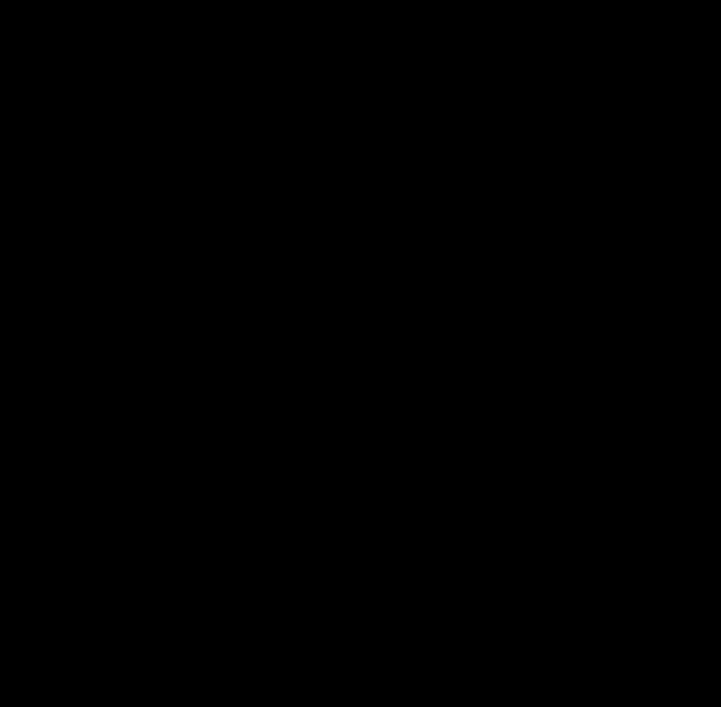

(b) Phase diagram

Figure 5. Endogenous bargaining power

distributed. In this case, the population with less decision power is better off when holding different preferences than the other population, and $\left(q_{t}^{m}, q_{t}^{f}\right)$ converges to an inegalitarian equilibrium. Alternatively, in country $\mathcal{B}$, the job priority preference always dominates and gender differences in preferences tend to vanish over time. This simple example illustrates that two countries, characterised by the same technological level, may converge towards a quite different situation in terms of gender differences in preferences.

The multiplicity of equilibria can also imply path dependency in the sense that initial technological differences may generate long-lasting discrepancies in preferences between genders. Assume now that in country $\mathcal{A}, \lambda$ is initially smaller than in country $\mathcal{B}$. A smaller $\lambda$ induces a larger value of $\hat{\delta}$ and a smaller size of the basins of attraction of inegalitarian equilibria in country $\mathcal{A}{ }^{24}$ As a consequence, even if the distribution of preferences is initially the same in both countries, in some cases, country $\mathcal{B}$ converges towards an inegalitarian equilibrium while country $\mathcal{A}$ ends up in the $(1,1)$ equilibrium. As these equilibria are locally stable, discrepancies between the two countries will perpetuate themselves even if they adopt the same technology afterwards.

This last result may be interestingly related to a recent paper by Alesina et al. (2013). The authors show that countries initially characterised by agricultural technologies relying heavily on physical strength (such as plough cultivation) are more likely to display inegalitarian gender stereotypes today. As a result, even if technological disparities in agriculture have now disappeared 24. To see this, note that $\tilde{\mu}_{1}(\lambda)$ is decreasing in $\lambda, \mu\left(\delta_{t}\right)$ is increasing in $\delta_{t}$ and $\hat{\delta}$ is defined as $\mu(\hat{\delta})=\tilde{\mu}_{1}(\lambda)$. 
among developed countries, views about gender roles may remain persistently different. In our set-up, these inegalitarian views are self-confirmed by persistent differences in preferences between men and women.

\section{Conclusion}

Recent findings in experimental economics stress the existence of culturally determined gender differences in preferences. These results have important consequences on our understanding of the evolution of gender inequalities. Indeed, these evolutions may be, at least partly, driven by changes in men and women's preferences. The aim of our article is to provide a tractable theoretical framework to model the dynamics of the gender gap in preferences. To this end, we extend the cultural transmission model proposed by Bisin and Verdier (2001) by considering that parents may choose to socialise their sons and their daughters differently. We are then able to characterise the joint dynamics of preferences within the population of men and women. Our framework may be used to analyse why some differences in preferences persist while others vanish over times. We provide a particular application focusing on the relative disutility associated with market work vs. housework. We show how technological or institutional changes, by transforming the way spouses interact within the household, may explain the evolution of gender differences in preferences.

Our model could be extended in several directions. Relaxing the assumption of random matching on the marriage market in particular would generate some new motives for parents to socialise their children. Indeed, they would have incentives to transmit cultural traits which are likely to improve the attractiveness of their children on the marriage market. Since these traits may be different for men and women, this effect could be an impediment to reducing the gender gap in preferences. The analysis of these mechanisms may constitute an interesting path for further research.

\section{Appendices}

\section{A Proof of Proposition 1}

Under the assumption of myopic expectations the cultural dynamics of the economy is described by the following two-dimensional dynamical system (see expression (14)):

$$
\left\{\begin{array}{l}
q_{t+1}^{m}=g^{m}\left(q_{t}^{m}, q_{t}^{f}\right) \\
q_{t+1}^{f}=g^{f}\left(q_{t}^{m}, q_{t}^{f}\right)
\end{array}\right.
$$


with

$$
g^{s}\left(q_{t}^{m}, q_{t}^{f}\right)=\left\{\begin{array}{lll}
q_{t}^{s}+\left(1-q_{t}^{s}\right)^{2} k\left[\left(B^{s}+A^{s}\right) q_{t}^{-s}-B^{s}\right] & \text { if } \quad\left(B^{s}+A^{s}\right) q_{t}^{-s} \geq B^{s} \\
q_{t}^{s}+\left(q_{t}^{s}\right)^{2} k\left[\left(B^{s}+A^{s}\right) q_{t}^{-s}-B^{s}\right] & \text { if } \quad\left(B^{s}+A^{s}\right) q_{t}^{-s}<B^{s}
\end{array}\right.
$$

Then:

i When $A^{s}>0$ and $B^{s}<0$, it is straightforward that $g^{s}\left(q_{t}^{m}, q_{t}^{f}\right)=q_{t}^{s}$ iff $q_{t}^{s}=1$ and $g^{s}\left(q_{t}^{m}, q_{t}^{f}\right)>$ $q_{t}^{s}$ for all values of $q_{t}^{s}<1$ and $q_{t}^{-s} \in[0,1]$.

ii When $A^{s}<0$ and $B^{s}>0$, it is straightforward that $g^{s}\left(q_{t}^{m}, q_{t}^{f}\right)=q_{t}^{s}$ iff $q_{t}^{s}=0$ and $g^{s}\left(q_{t}^{m}, q_{t}^{f}\right)<$ $q_{t}^{s}$ for all values of $q_{t}^{s}>0$ and $q_{t}^{-s} \in[0,1]$.

iii When $A^{s}>0$ and $B^{s}>0$, the unique values of $q_{t}^{s}$ such that $g^{s}\left(q_{t}^{m}, q_{t}^{f}\right)=q_{t}^{s}$ are:

$$
\left\{\begin{array}{llll}
q_{t}^{s}=1 & \text { if } & q_{t}^{-s}>\bar{q}^{s} \\
q_{t}^{s}=0 & \text { if } & q_{t}^{-s}<\bar{q}^{s} \\
q_{t}^{s} \in[0,1] & \text { if } & q_{t}^{-s}=\bar{q}^{s}
\end{array}\right.
$$

Moreover

$$
g^{s}\left(q_{t}^{m}, q_{t}^{f}\right)\left\{\begin{array}{l}
\geq \\
\leq
\end{array}\right\} q_{t}^{s} \quad \text { if } \quad q_{t}^{-s}\left\{\begin{array}{l}
\geq \\
\leq
\end{array}\right\} \bar{q}^{s}
$$

iv When $A^{s}<0$ and $B^{s}<0$, the unique values of $q_{t}^{s}$ such that $g^{s}\left(q_{t}^{m}, q_{t}^{f}\right)=q_{t}^{s}$ are:

$$
\left\{\begin{array}{lll}
q_{t}^{s}=1 & \text { if } & q_{t}^{-s}<\bar{q}^{s} \\
q_{t}^{s}=0 & \text { if } & q_{t}^{-s}>\bar{q}^{s} \\
q_{t}^{s} \in[0,1] & \text { if } & q_{t}^{-s}=\bar{q}^{s}
\end{array}\right.
$$

Moreover

$$
g^{s}\left(q_{t}^{m}, q_{t}^{f}\right)\left\{\begin{array}{l}
\geq \\
\leq
\end{array}\right\} q_{t}^{s} \quad \text { if } \quad q_{t}^{-s}\left\{\begin{array}{l}
\leq \\
\geq
\end{array}\right\} \bar{q}^{s}
$$

Steady state equilibria of the dynamical system (A.1) are pairs $\left(\tilde{q}^{m}, \tilde{q}^{f}\right)$ such that:

$$
\left\{\begin{array}{l}
\tilde{q}^{m}=g^{m}\left(\tilde{q}^{m}, \tilde{q}^{f}\right) \\
\tilde{q}^{f}=g^{f}\left(\tilde{q}^{m}, \tilde{q}^{f}\right)
\end{array}\right.
$$

From the previous analysis (points i to iv) we deduce the steady state equilibria in all the configurations listed in the Proposition: 


\begin{tabular}{|c|c|c|c|c|c|}
\hline \multicolumn{2}{|c|}{} & \multicolumn{2}{|c|}{$A^{f}>0$} & \multicolumn{2}{c|}{$A^{f}<0$} \\
\cline { 3 - 6 } \multicolumn{2}{|c|}{} & $A^{m}>0$ & $A^{m}<0$ & $A^{m}>0$ & $A^{m}<0$ \\
\hline \multirow{2}{*}{$B^{f}>0$} & $B^{m}>0$ & $(0,0),(1,1),\left(\bar{q}^{m}, \bar{q}^{f}\right)$ & $(0,0)$ & $(0,0)$ & $(0,0)$ \\
\cline { 2 - 6 } & $B^{m}<0$ & $(1,1)$ & $\left(\bar{q}^{m}, \bar{q}^{f}\right)$ & $(1,0)$ & $(1,0)$ \\
\hline \multirow{2}{*}{$B^{f}<0$} & $B^{m}>0$ & $(1,1)$ & $(0,1)$ & $\left(\bar{q}^{m}, \bar{q}^{f}\right)$ & $(0,1)$ \\
\cline { 2 - 6 } & $B^{m}<0$ & $(1,1)$ & $(0,1)$ & $(1,0)$ & $(0,1),(1,0),\left(\bar{q}^{m}, \bar{q}^{f}\right)$ \\
\hline
\end{tabular}

Table 1 - Steady state equilbria

It remains to verify that $\left(\bar{q}^{m}, \bar{q}^{f}\right)$ constitutes a saddle point when $A^{f}>0, B^{f}>0, A^{m}>0, B^{m}>$ 0 and $A^{f}<0, B^{f}<0, A^{m}<0, B^{m}<0$ and is a spiral source when $A^{f}>0, B^{f}>0, A^{m}<0, B^{m}<0$ and $A^{f}<0, B^{f}<0, A^{m}>0, B^{m}>0$. To this end we study the local dynamics of the economy around the steady state $\left(\bar{q}^{m}, \bar{q}^{f}\right)$ (see Galor 2007).

Let us define the Jacobian matrix associated with the fixed point $\left(\bar{q}^{m}, \bar{q}^{f}\right)$ of the dynamical system (A.1):

$$
\mathcal{J}=\left(\begin{array}{ll}
\frac{\partial g^{f}}{\partial q_{t}^{f}}\left(\bar{q}^{m}, \bar{q}^{f}\right) & \frac{\partial g^{f}}{\partial q_{t}^{m}}\left(\bar{q}^{m}, \bar{q}^{f}\right) \\
\frac{\partial g^{m}}{\partial q_{t}^{f}}\left(\bar{q}^{m}, \bar{q}^{f}\right) & \frac{\partial g^{m}}{\partial q_{t}^{m}}\left(\bar{q}^{m}, \bar{q}^{f}\right)
\end{array}\right)
$$

To determine the stability type of $\left(\bar{q}^{m}, \bar{q}^{f}\right)$ we have to compute the trace and the determinant of $\mathcal{J}$ (respectively denoted by $\operatorname{Tr}(\mathcal{J})$ and $\operatorname{Det}(\mathcal{J})$ ). We have:

$$
\frac{\partial g^{f}}{\partial q_{t}^{f}}\left(\bar{q}^{m}, \bar{q}^{f}\right)=\frac{\partial g^{m}}{\partial q_{t}^{m}}\left(\bar{q}^{m}, \bar{q}^{f}\right)=1
$$

and then $\operatorname{Tr}(\mathcal{J})=2$. Thus, if $|1+\operatorname{Det}(\mathcal{J})|<2,\left(\bar{q}^{m}, \bar{q}^{f}\right)$ is a saddle; and if $|1+\operatorname{Det}(\mathcal{J})|>2$ (which implies that $\left.|\operatorname{Det}(\mathcal{J})|>\operatorname{Tr}(\mathcal{J})^{2} / 4=1\right),\left(\bar{q}^{m}, \bar{q}^{f}\right)$ is a source. Moreover:

$$
\frac{\partial g^{s}}{\partial q_{t}^{-s}}\left(\bar{q}^{m}, \bar{q}^{f}\right)=\left\{\begin{array}{lll}
\frac{k\left(A^{s}\right)^{2}}{B^{s}+A^{s}} & \text { if } & \left(B^{s}+A^{s}\right) \bar{q}^{-s} \geq B^{s} \\
\frac{k\left(B^{s}\right)^{2}}{B^{s}+A^{s}} & \text { if } & \left(B^{s}+A^{s}\right) \bar{q}^{-s}<B^{s}
\end{array}\right.
$$

and

$$
\operatorname{Det}(\mathcal{J})=1-\frac{\partial g^{f}}{\partial q_{t}^{m}}\left(\bar{q}^{m}, \bar{q}^{f}\right) \cdot \frac{\partial g^{m}}{\partial q_{t}^{f}}\left(\bar{q}^{m}, \bar{q}^{f}\right)
$$

Note that, Assumption 1 ensures that $\operatorname{Det}(\mathcal{J})$ is always positive. Then, using expression (A.2) we get that:

$$
\operatorname{Det}(\mathcal{J})\left\{\begin{array}{c}
< \\
>
\end{array}\right\} 1 \quad \text { if } \quad\left(A^{f}+B^{f}\right)\left(A^{m}+B^{m}\right)\left\{\begin{array}{l}
> \\
<
\end{array}\right\} 0
$$

It directly follows that $\left(\bar{q}^{m}, \bar{q}^{f}\right)$ is a saddle for $A^{f}>0, B^{f}>0, A^{m}>0, B^{m}>0$ and $A^{f}<0, B^{f}<$ $0, A^{m}<0, B^{m}<0$ and is a source when $A^{f}>0, B^{f}>0, A^{m}<0, B^{m}<0$ and $A^{f}<0, B^{f}<$ 
$0, A^{m}>0, B^{m}>0$.

\section{B Proof of Proposition 2}

Let us first introduce some materials which proves to be useful in the proof of the proposition. In particular, let us define the two functions:

$$
\begin{gathered}
\Psi(q)=\frac{B-(B+A) q+k(B+A) B(1-q)^{2}}{k(B+A)^{2}(1-q)^{2}} \\
\Theta(q)=\frac{B+k B(B+A)(q)^{2}-(B+A) q}{k(B+A)^{2}(q)^{2}}
\end{gathered}
$$

and the four sets:

$$
\begin{aligned}
& \boldsymbol{\Omega}_{\mathbf{1}} \equiv\left\{\left(q_{t}^{m}, q_{t}^{f}\right) \in[0,1]^{2}: q_{t}^{m} \geq \Psi\left(q_{t}^{f}\right) \quad \text { and } \quad q_{t}^{f} \geq \Psi\left(q_{t}^{m}\right)\right\} \\
& \boldsymbol{\Omega}_{\mathbf{2}} \equiv\left\{\left(q_{t}^{m}, q_{t}^{f}\right) \in[0,1]^{2}: q_{t}^{m}<\Theta\left(q_{t}^{f}\right) \quad \text { and } \quad q_{t}^{f}<\Theta\left(q_{t}^{m}\right)\right\} \\
& \boldsymbol{\Omega}_{\mathbf{3}} \equiv\left\{\left(q_{t}^{m}, q_{t}^{f}\right) \in[0,1]^{2}: q_{t}^{m} \geq \Theta\left(q_{t}^{f}\right) \quad \text { and } \quad q_{t}^{f}<\Psi\left(q_{t}^{m}\right)\right\} \\
& \boldsymbol{\Omega}_{\mathbf{4}} \equiv\left\{\left(q_{t}^{m}, q_{t}^{f}\right) \in[0,1]^{2}: q_{t}^{m}<\Psi\left(q_{t}^{f}\right) \quad \text { and } \quad q_{t}^{f} \geq \Theta\left(q_{t}^{m}\right)\right\}
\end{aligned}
$$

As states in Lemma 1 , each $\boldsymbol{\Omega}_{\mathbf{i}}$ represents a set of value of $\left(q_{t}^{m}, q_{t}^{f}\right)$ such that one particular expectation is admissible.

Lemma 1 Consider that expectations are perfect, both $A$ and $B$ are negative and Assumption 1 and condition (16) hold:

$i$ For each $\left(q_{t}^{m}, q_{t}^{f}\right) \in \mathbf{\Omega}_{\mathbf{1}}$, there is a unique self-fulfilling expectation: $\left(\hat{q}_{t+1}^{m}, \hat{q}_{t+1}^{f}\right) \in(0, \bar{q}]^{2}$.

ii For each $\left(q_{t}^{m}, q_{t}^{f}\right) \in \mathbf{\Omega}_{\mathbf{2}}$, there is a unique self-fulfilling expectation: $\left(\hat{q}_{t+1}^{m}, \hat{q}_{t+1}^{f}\right) \in(\bar{q}, 1)^{2}$.

iii For each $\left(q_{t}^{m}, q_{t}^{f}\right) \in \boldsymbol{\Omega}_{\mathbf{3}} \backslash \boldsymbol{\Omega}_{\mathbf{4}}$, there is a unique self-fulfilling expectation: $\left(\hat{q}_{t+1}^{m}, \hat{q}_{t+1}^{f}\right) \in(\bar{q}, 1) \times$ $(0, \bar{q}]$.

iv For each $\left(q_{t}^{m}, q_{t}^{f}\right) \in \mathbf{\Omega}_{\mathbf{4}} \backslash \boldsymbol{\Omega}_{\mathbf{3}}$, there is a unique self-fulfilling expectation: $\left(\hat{q}_{t+1}^{m}, \hat{q}_{t+1}^{f}\right) \in(0, \bar{q}] \times$ $(\bar{q}, 1)$.

$v$ For each $\left(q_{t}^{m}, q_{t}^{f}\right) \in \mathbf{\Omega}_{\mathbf{4}} \cap \boldsymbol{\Omega}_{\mathbf{3}}$, there is two self-fulfilling expectations: $\left(\hat{q}_{t+1}^{m}, \hat{q}_{t+1}^{f}\right) \in(0, \bar{q}] \times(\bar{q}, 1)$ and $\left(\hat{q}_{t+1}^{m}, \hat{q}_{t+1}^{f}\right) \in(\bar{q}, 1) \times(0, \bar{q}]$. 
Proof. Under the assumption of perfect expectations the cultural dynamics of the economy is described by the following two-dimensional dynamical system (see expression (14)):

$$
\left\{\begin{array}{l}
q_{t+1}^{m}=h^{m}\left(q_{t}^{m}, q_{t}^{f}\right) \\
q_{t+1}^{f}=h^{f}\left(q_{t}^{m}, q_{t}^{f}\right)
\end{array}\right.
$$

with

$$
h^{s}\left(q_{t}^{m}, q_{t}^{f}\right)=\left\{\begin{array}{lll}
h_{1}^{s}\left(q_{t}^{m}, q_{t}^{f}\right) & \text { if } \quad(B+A) q_{t+1}^{s} \geq B \text { and }(B+A) q_{t+1}^{-s} \geq B \\
h_{2}^{s}\left(q_{t}^{m}, q_{t}^{f}\right) & \text { if } \quad(B+A) q_{t+1}^{s}<B \text { and }(B+A) q_{t+1}^{-s}<B \\
h_{3}^{s}\left(q_{t}^{m}, q_{t}^{f}\right) & \text { if } \quad(B+A) q_{t+1}^{s} \geq B \text { and }(B+A) q_{t+1}^{-s}<B \\
h_{4}^{s}\left(q_{t}^{m}, q_{t}^{f}\right) & \text { if } \quad(B+A) q_{t+1}^{s}<B \text { and }(B+A) q_{t+1}^{-s} \geq B
\end{array}\right.
$$

and

$$
\begin{aligned}
h_{1}^{s}\left(q_{t}^{m}, q_{t}^{f}\right) & =\frac{q_{t}^{s}+\left(1-q_{t}^{s}\right)^{2} k\left[(B+A) q_{t}^{-s}-B-k B(B+A)\left(1-q_{t}^{-s}\right)^{2}\right]}{1-k^{2}\left(1-q_{t}^{s}\right)^{2}\left(1-q_{t}^{-s}\right)^{2}(B+A)^{2}} \\
h_{2}^{s}\left(q_{t}^{m}, q_{t}^{f}\right) & =\frac{q_{t}^{s}+\left(q_{t}^{s}\right)^{2} k\left[(B+A) q_{t}^{-s}-B-k B(B+A)\left(q_{t}^{-s}\right)^{2}\right]}{1-k^{2}\left(q_{t}^{s}\right)^{2}\left(q_{t}^{-s}\right)^{2}(B+A)^{2}} \\
h_{3}^{s}\left(q_{t}^{m}, q_{t}^{f}\right) & =\frac{q_{t}^{s}+\left(q_{t}^{s}\right)^{2} k\left[(B+A) q_{t}^{-s}-B-k B(B+A)\left(1-q_{t}^{-s}\right)^{2}\right]}{1-k^{2}\left(1-q_{t}^{-s}\right)^{2}\left(q_{t}^{s}\right)^{2}(B+A)^{2}} \\
h_{4}^{s}\left(q_{t}^{m}, q_{t}^{f}\right) & =\frac{q_{t}^{s}+\left(1-q_{t}^{s}\right)^{2} k\left[(B+A) q_{t}^{-s}-B-k B(B+A)\left(q_{t}^{-s}\right)^{2}\right]}{1-k^{2}\left(1-q_{t}^{s}\right)^{2}\left(q_{t}^{-s}\right)^{2}(B+A)^{2}}
\end{aligned}
$$

We can now determine in which configuration one expectation is self-fulfilling:

i The condition $\left(\hat{q}_{t+1}^{m}, \hat{q}_{t+1}^{f}\right) \in(0, \bar{q}]^{2}$ translates into $(B+A) \hat{q}_{t+1}^{f} \geq B$ and $(B+A) \hat{q}_{t+1}^{m} \geq B$ such that the perfect foresight dynamics of preferences is given by $q_{t+1}^{m}=h_{1}^{m}\left(q_{t}^{m}, q_{t}^{f}\right)$ and $q_{t+1}^{f}=h_{1}^{f}\left(q_{t}^{m}, q_{t}^{f}\right)$. It is easy to verify that $h_{1}^{m}\left(q_{t}^{m}, q_{t}^{f}\right) \leq \bar{q}\left(\operatorname{resp} . h_{1}^{f}\left(q_{t}^{m}, q_{t}^{f}\right) \leq \bar{q}\right)$ if and only if $q_{t}^{f} \geq \Psi\left(q_{t}^{m}\right)$ (resp. $q_{t}^{m} \geq \Psi\left(q_{t}^{f}\right)$ ) which implies $\left(q_{t}^{m}, q_{t}^{f}\right) \in \mathbf{\Omega}_{\mathbf{1}}$.

ii The condition $\left(\hat{q}_{t+1}^{m}, \hat{q}_{t+1}^{f}\right) \in(\bar{q}, 1)^{2}$ translates into $(B+A) \hat{q}_{t+1}^{f}<B$ and $(B+A) \hat{q}_{t+1}^{m}<B$ such that the perfect foresight dynamics of preferences is given by $q_{t+1}^{m}=h_{2}^{m}\left(q_{t}^{m}, q_{t}^{f}\right)$ and $q_{t+1}^{f}=h_{2}^{f}\left(q_{t}^{m}, q_{t}^{f}\right)$. It is easy to verify that $h_{2}^{m}\left(q_{t}^{m}, q_{t}^{f}\right)>\bar{q}\left(\operatorname{resp} . h_{2}^{f}\left(q_{t}^{m}, q_{t}^{f}\right)>\bar{q}\right)$ if and only if $q_{t}^{f}<\Theta\left(q_{t}^{m}\right)$ (resp. $\left.q_{t}^{m}<\Theta\left(q_{t}^{f}\right)\right)$ which implies $\left(q_{t}^{m}, q_{t}^{f}\right) \in \mathbf{\Omega}_{\mathbf{2}}$.

iii The condition $\left(\hat{q}_{t+1}^{m}, \hat{q}_{t+1}^{f}\right) \in(0, \bar{q}] \times(\bar{q}, 1)$ translates into $(B+A) \hat{q}_{t+1}^{f} \geq B$ and $(B+A) \hat{q}_{t+1}^{m}<B$ such that the perfect foresight dynamics of preferences is given by $q_{t+1}^{m}=h_{4}^{m}\left(q_{t}^{m}, q_{t}^{f}\right)$ and $q_{t+1}^{f}=h_{3}^{f}\left(q_{t}^{m}, q_{t}^{f}\right)$. It is easy to verify that $h_{3}^{f}\left(q_{t}^{m}, q_{t}^{f}\right) \leq \bar{q}\left(\right.$ resp. $\left.h_{4}^{m}\left(q_{t}^{m}, q_{t}^{f}\right)>\bar{q}\right)$ if and only if $q_{t}^{m} \geq \Theta\left(q_{t}^{f}\right)$ (resp. $\left.q_{t}^{f}<\Psi\left(q_{t}^{m}\right)\right)$ which implies $\left(q_{t}^{m}, q_{t}^{f}\right) \in \boldsymbol{\Omega}_{\mathbf{3}}$.

iv The condition $\left(\hat{q}_{t+1}^{m}, \hat{q}_{t+1}^{f}\right) \in(\bar{q}, 1) \times(0, \bar{q}]$ translates into $(B+A) \hat{q}_{t+1}^{f}<B$ and $(B+A) \hat{q}_{t+1}^{m} \geq B$ such that the perfect foresight dynamics of preferences is given by $q_{t+1}^{m}=h_{3}^{m}\left(q_{t}^{m}, q_{t}^{f}\right)$ and 
$q_{t+1}^{f}=h_{4}^{f}\left(q_{t}^{m}, q_{t}^{f}\right)$. It is easy to verify that $h_{4}^{f}\left(q_{t}^{m}, q_{t}^{f}\right)>\bar{q}\left(\operatorname{resp} . h_{3}^{m}\left(q_{t}^{m}, q_{t}^{f}\right) \leq \bar{q}\right)$ if and only if $q_{t}^{m}<\Psi\left(q_{t}^{f}\right)\left(\right.$ resp. $\left.q_{t}^{f} \geq \Theta\left(q_{t}^{m}\right)\right)$ which implies $\left(q_{t}^{m}, q_{t}^{f}\right) \in \mathbf{\Omega}_{\mathbf{4}}$.

The results of the Lemma directly follow.

Let us now represent $\boldsymbol{\Omega}_{\mathbf{1}}, \boldsymbol{\Omega}_{\mathbf{2}}, \boldsymbol{\Omega}_{\mathbf{3}}$ and $\boldsymbol{\Omega}_{\mathbf{4}}$ in the space state $\left(q_{t}^{m}, q_{t}^{f}\right)$ when $B<A .{ }^{25}$ Note that

$$
\frac{\partial \Psi(q)}{\partial q}=\frac{B-A-q(B+A)}{k(B+A)^{2}(1-q)^{3}}
$$

Then, since $B<A<0, \Psi(q)$ is U-shaped, reaching a minimum for $q=\frac{B-A}{A+B} \in(0,1)$. Moreover:

$$
\frac{\partial \Theta(q)}{\partial q}=\frac{(B+A) q-2 B}{k(B+A)^{2} q^{3}}
$$

Then, $\Theta(q)$ is increasing on $[0,1]$. Moreover, we can verify that:

$$
\Psi\left(\frac{1}{2}\right)=\Theta\left(\frac{1}{2}\right) \quad \text { and } \quad \Psi(\bar{q})=\Theta(\bar{q})=\bar{q} \in(0,1)
$$

Such that the curves $\Psi\left(q_{t}^{m}\right), \Theta\left(q_{t}^{m}\right), \Psi\left(q_{t}^{f}\right)$ and $\Theta\left(q_{t}^{f}\right)$ cross at the point $(\bar{q}, \bar{q})$. Moreover:

$$
\begin{aligned}
& \Psi(0)=\bar{q}+\frac{B}{k(B+A)^{2}} \quad \text { and } \quad \lim _{q \rightarrow 1^{-}} \Psi(q)=+\infty \\
& \Theta(1)=\bar{q}-\frac{A}{k(B+A)^{2}} \quad \text { and } \quad \lim _{q \rightarrow 0^{+}} \Theta(q)=-\infty
\end{aligned}
$$

Using condition (16) it is easy to verify that $\Psi(0) \in(0, \bar{q})$ and $\Theta(1)<\in(\bar{q}, 1)$. Those properties allow us to draw the curves $\Psi\left(q_{t}^{m}\right)$ and $\Theta\left(q_{t}^{m}\right)$ in the plan $\left(q_{t}^{m}, q_{t}^{f}\right)$ and by symmetry the curves $\Psi\left(q_{t}^{f}\right)$ and $\Theta\left(q_{t}^{f}\right)$. Then, by definition of $\boldsymbol{\Omega}_{\mathbf{1}}, \boldsymbol{\Omega}_{\mathbf{2}}, \boldsymbol{\Omega}_{\mathbf{3}}$ and $\boldsymbol{\Omega}_{\mathbf{4}}$, we derive Figure 6 and deduce that the set $I=\boldsymbol{\Omega}_{\mathbf{3}} \cap \boldsymbol{\Omega}_{\mathbf{4}}$ is non empty.

We can now prove the Proposition. Using expressions (B.5)-(B.8) and after some algebra we conclude that:

- If $(B+A) q_{t+1}^{s} \geq B$ and $(B+A) q_{t+1}^{-s} \geq B, q_{t+1}^{s}>(<) q_{t}^{s} \Leftrightarrow q_{t}^{s}>(<) \Psi\left(q_{t}^{-s}\right)$.

- If $(B+A) q_{t+1}^{s}<B$ and $(B+A) q_{t+1}^{-s}<B, q_{t+1}^{s}>(<) q_{t}^{s} \Leftrightarrow q_{t}^{s}>(<) \Theta\left(q_{t}^{-s}\right)$.

- If $(B+A) q_{t+1}^{s} \geq B$ and $(B+A) q_{t+1}^{-s}<B, q_{t+1}^{s}>(<) q_{t}^{s} \Leftrightarrow q_{t}^{s}>(<) \Psi\left(q_{t}^{-s}\right)$.

- If $(B+A) q_{t+1}^{s}<B$ and $(B+A) q_{t+1}^{-s} \geq B, q_{t+1}^{s}>(<) q_{t}^{s} \Leftrightarrow q_{t}^{s}>(<) \Theta\left(q_{t}^{-s}\right)$.

Then, by the points i. to iv. of the proof of Lemma 1: when $\left(q_{t}^{m}, q_{t}^{f}\right) \in \boldsymbol{\Omega}_{\mathbf{1}}$, both $q_{t}^{f}$ and $q_{t}^{m}$ increase over time; when $\left(q_{t}^{m}, q_{t}^{f}\right) \in \boldsymbol{\Omega}_{\mathbf{2}}$, both $q_{t}^{f}$ and $q_{t}^{m}$ decrease over time; when $\left(q_{t}^{m}, q_{t}^{f}\right) \in \boldsymbol{\Omega}_{\mathbf{3}} \backslash \boldsymbol{\Omega}_{\mathbf{4}}, q_{t}^{f}$

25. This assumption is made without loss of generality. Symmetric results will be obtain by considering $A<B$. 


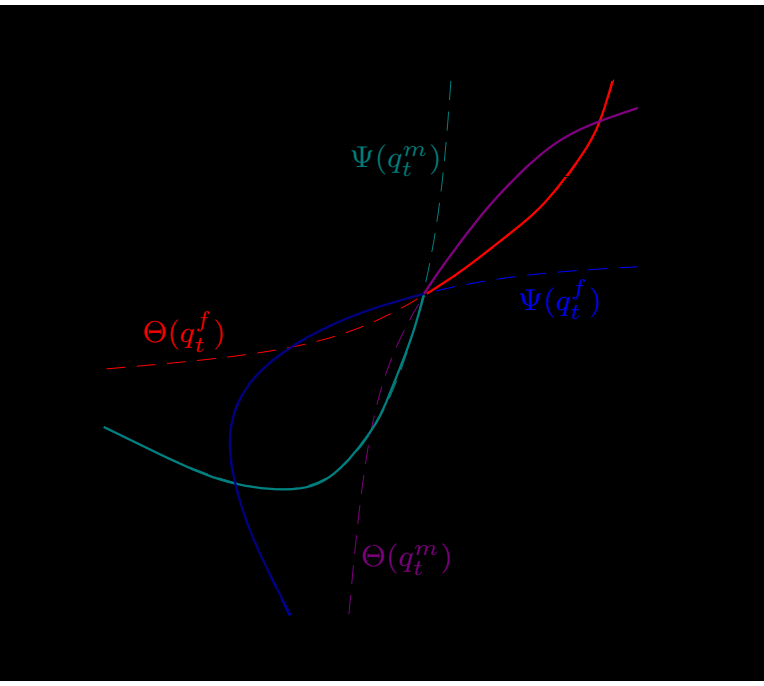

Figure 6. Partition of the state space for $B<A<0$

decreases while $q_{t}^{m}$ increases over time; when $\left(q_{t}^{m}, q_{t}^{f}\right) \in \boldsymbol{\Omega}_{\mathbf{4}} \backslash \boldsymbol{\Omega}_{\mathbf{3}}, q_{t}^{f}$ increases while $q_{t}^{m}$ decreases over time.

We have now to verify if $(\bar{q}, \bar{q})$ constitutes a saddle point. As in the proof of Proposition 1 , we have to compute the trace and the determinant of the jacobian matrix

$$
\mathcal{J}=\left(\begin{array}{cc}
\frac{\partial h^{f}}{\partial q_{t}^{f}}\left(\bar{q}^{m}, \bar{q}^{f}\right) & \frac{\partial h^{f}}{\partial q_{t}^{m}}\left(\bar{q}^{m}, \bar{q}^{f}\right) \\
\frac{\partial h^{m}}{\partial q_{t}^{f}}\left(\bar{q}^{m}, \bar{q}^{f}\right) & \frac{\partial h^{m}}{\partial q_{t}^{m}}\left(\bar{q}^{m}, \bar{q}^{f}\right)
\end{array}\right)
$$

in each region of the neighborhood of $(\bar{q}, \bar{q})$. Using the proof of Lemma 1 and after some algebra we obtain:

$$
\operatorname{Tr}(\mathcal{J})=\left\{\begin{array}{lll}
\frac{2(B+A)^{2}}{(B+A)^{2}-k^{2} A^{4}} & \text { if } \quad\left(q_{t}^{m}, q_{t}^{f}\right) \in \boldsymbol{\Omega}_{\mathbf{1}} \\
\frac{2(B+A)^{2}}{(B+A)^{2}-k^{2} B^{4}} & \text { if } \quad\left(q_{t}^{m}, q_{t}^{f}\right) \in \boldsymbol{\Omega}_{\mathbf{2}} \\
\frac{2(B+A)^{2}}{(B+A)^{2}-k^{2} A^{2} B^{2}} & \text { if } \quad\left(q_{t}^{m}, q_{t}^{f}\right) \in \boldsymbol{\Omega}_{\mathbf{3}} \backslash \boldsymbol{\Omega}_{\mathbf{4}} \\
\frac{2(B+A)^{2}}{(B+A)^{2}-k^{2} A^{2} B^{2}} & \text { if } \quad\left(q_{t}^{m}, q_{t}^{f}\right) \in \boldsymbol{\Omega}_{\mathbf{4}} \backslash \boldsymbol{\Omega}_{\mathbf{3}}
\end{array}\right.
$$

and

$$
\operatorname{Det}(\mathcal{J})=\operatorname{Tr}(\mathcal{J}) / 2
$$

Both $\operatorname{Tr}(\mathcal{J})$ and $\operatorname{Det}(\mathcal{J})$ are positive since $k^{2}<\min \left\{1 / A^{2}, 1 / B^{2}\right\}$. Moreover, since $\operatorname{Det}(\mathcal{J})>1$, we have $\operatorname{Tr}(\mathcal{J})>\operatorname{Det}(\mathcal{J})+1$.

The last point of the proposition is directly derived from the point v. of Lemma 1. Indeed, $(0, \bar{q}] \times(\bar{q}, 1) \subset \boldsymbol{\Omega}_{\mathbf{3}} \backslash \boldsymbol{\Omega}_{\mathbf{4}}$ and $(\bar{q}, 1) \times(0, \bar{q}] \subset \boldsymbol{\Omega}_{\mathbf{4}} \backslash \boldsymbol{\Omega}_{\mathbf{3}}$. 


\section{Endogenous bargaining power - Derivation of the phase diagram}

- $q_{t}^{f} \in\left[q_{t}^{m}-\hat{\delta}, q_{t}^{m}+\hat{\delta}\right]:$

Preference $a$ dominates within both populations such that $q_{t}^{m}$ and $q_{t}^{f}$ increase over time.

- $q_{t}^{f}<q_{t}^{m}-\hat{\delta}$ :

Preference $a$ dominates within the population of males, such that $q_{t}^{m}$ increases over time; while preferences display asymmetric complementarity within the population of females. Then, the stationary locus of $q_{t}^{f}$ is given by $q_{t}^{m}=B^{f} /\left(A^{f}+B^{f}\right)$. However, $A^{f}$ and $B^{f}$ depend on $\mu$ which, in turn, may be expressed as a function of $q_{t}^{m}-q_{t}^{f}$. Let us define $\bar{q}^{f}\left(q_{t}^{m}\right)$ as the stationary locus of $q_{t}^{f}$ in the plan $\left(q_{t}^{m}, q_{t}^{f}\right)$. By (26), this locus may be implicitly characterized by the following equation:

$$
q_{t}^{m}=\frac{(2-\lambda)^{2}\left[\mu\left(q_{t}^{m}-q_{t}^{f}\right)\right]^{2}}{\left[(2-\lambda)^{2}+\lambda^{2}\right]\left[\mu\left(q_{t}^{m}-q_{t}^{f}\right)\right]^{2}-(1-\lambda)^{2}}
$$

This equation may be rewritten as:

$$
\mu\left(q_{t}^{m}-q_{t}^{f}\right)=\sqrt{\Gamma\left(q_{t}^{m}\right)} \quad \text { with } \quad \Gamma\left(q_{t}^{m}\right) \equiv \frac{q_{t}^{m}(1-\lambda)^{2}}{\left[(2-\lambda)^{2}+\lambda^{2}\right] q_{t}^{m}-(2-\lambda)^{2}}
$$

By differentiating (C.2) we get that

$$
\frac{d q_{t}^{f}}{d q_{t}^{m}}=1-\frac{\Gamma^{\prime}\left(q_{t}^{m}\right)}{2 \mu^{\prime}\left(q_{t}^{m}-q_{t}^{f}\right) \sqrt{\Gamma\left(q_{t}^{m}\right)}}>0
$$

The positivity of $d q_{t}^{f} / d q_{t}^{m}$ directly comes from the positivity of $\mu^{\prime}\left(q_{t}^{m}-q_{t}^{f}\right)$ and the negativity of $\Gamma^{\prime}\left(q_{t}^{m}\right)$. It implies that the stationary locus of $q_{t}^{f}$ is upward slopping in the plan $\left(q_{t}^{m}, q_{t}^{f}\right)$, i.e. $\bar{q}^{f^{\prime}}\left(q_{t}^{m}\right)>0$. Moreover, $\sqrt{\Gamma(1)}=(1-\lambda) / \lambda=\tilde{\mu}_{1}(\lambda)$ such that expression (C.2) for $q_{t}^{m}=1$ implies that:

$$
\mu\left(1-q_{t}^{f}\right)=\tilde{\mu}_{1}(\lambda) \quad \Leftrightarrow \quad \bar{q}^{f}(1)=1-\hat{\delta}
$$

Let us now derive the threshold value $\tilde{q}^{m}$ of $q_{t}^{m}$ such that $\bar{q}^{f}\left(\tilde{q}^{m}\right)=0$. This value is solution of the equation $\mu\left(\tilde{q}^{m}\right)=\sqrt{\Gamma\left(\tilde{q}^{m}\right)}$. Since, $\mu\left(q_{t}^{m}\right)$ is increasing on $[0,1]$ with $\mu(0)=1 / 2$ and $\mu(1)=\bar{\mu}$ and $\Gamma\left(q_{t}^{m}\right)$ is decreasing on $[0,1]$, have a vertical asymptote at $q_{t}^{m}=(2-\lambda)^{2} /\left[(2-\lambda)^{2}+\lambda^{2}\right] \in(0,1)$ and $\Gamma(1)=\tilde{\mu}_{1}(\lambda)<\bar{\mu}$, the solution $\tilde{q}^{m}$ is unique. Moreover, $\mu\left(q^{m}\right)>\tilde{\mu}_{1}(\lambda)$ implies that $\tilde{q}^{m}>\hat{\delta}$.

Finally, from the proof of Proposition 1, we deduce that, $q_{t}^{f}$ increases over time when $q_{t}^{m}<$ $\bar{q}^{f}\left(q_{t}^{m}\right)$ and $q_{t}^{f}$ decreases over time when $q_{t}^{m}>\bar{q}^{f}\left(q_{t}^{m}\right)$.

- $q_{t}^{f}<q_{t}^{m}-\hat{\delta}$ : 
Preference $a$ dominates within the population of females, such that $q_{t}^{f}$ increases over time; while preferences display asymmetric complementarity within the population of males. The stationary locus of $q_{t}^{m}$, denoted by $\bar{q}^{m}\left(q_{t}^{f}\right)$ is constructed by symmetry of $\bar{q}^{f}\left(q_{t}^{m}\right)$. Moreover, from the proof of Proposition 1, we deduce that, $q_{t}^{m}$ increases over time when $q_{t}^{f}<\bar{q}^{m}\left(q_{t}^{m}\right)$ and $q_{t}^{m}$ decreases over time when $q_{t}^{f}>\bar{q}^{m}\left(q_{t}^{m}\right)$.

\section{References}

Alesina, A., Giuiliano, P. \& Nunn, N. 2013. On the Origins of Gender Roles: Women and the Plough. Quarterly Journal of Economics, 128, 469-530.

Andersen, S., Ertac, S., Gneezy, U., List, J.A. \& Maximiano, S. 2013. Gender, Competitiveness and Socialization at a Young Age: Evidence from a Matrilineal and a Patriarchal Society. Review of Economics and Statistics, 95, 1438-1443.

Basu, K. 2006. Gender and say: A model of household behaviour with endogenously determined balance of power. The Economic Journal, 116, 558-580.

Batesup, H.S., Booth, A., Shirtcliff, E.A., \& Granger, D.A. 2002. Testosterone, corsitol, and women's competition. Evolution of Human Behavior, 23, 181-192.

Bertrand, M., \& Hallock, K.F. 2001. The gender gap in top corporate jobs. Industrial and Labor Relations Review, 55, 3-21.

Bertrand, M., Goldin, C., \& Katz, L.F. 2010. Dynamics of the gender gap for young professionals in the financial and corporate sectors. American Economic Journal: Applied Economics, 2, 228-255.

Bianchi, S. 2012. Family Change and Time Allocation in American Families. The ANNALS of the American Academy of Political and Social Science, 638, 21-44.

Bisin, A., \& Verdier, T. 2001. The economics of cultural transmission and the evolution of preferences. Journal of Economic Theory, 97, 298-319.

Bisin, A., \& Verdier, T. 2011. The Economics of Cultural Transmission and Socialization. In: Jess Benhabib, Alberto Bisin, Matt Jackson (ed),Handbook of Social Economics. Elsevier.

Bricheno, P., \& Thornton, M. 2007. Role model, hero or champion? Children's views concerning role models. Educational Research, 49(4).

Bosquet, C., Combe, P.-P. \& García-Penãlosa, C. 2013. Gender and Competition: Evidence from Academic Promotions in France. CEPR Discussion Paper 9711.

Buser, T., Niederle, M. \& Oosterbeek, H. 2014. Gender, Competitiveness and Career Choices. Quarterly Journal of Economics, doi: 10.1093/qje/qju009. 
Croson, E., \& Gneezy, U. 2009. Gender differences in preferences. Journal of Economic Literature, 97, 298-319.

Doepke, M., \& Zilibotti, F. , L. 2008. Occupational choice and the spirit of capitalism. Quarterly Journal of Economics, 123, 747-793.

Doepke, M., \& Tertilt, M. 2009. Women's Liberation: What's in It for Men?. Quarterly Journal of Economics, 124, 1541-1591.

Dolado, J.J., García-Penãlosa, C. \& de la Rica, S. 2013 On gender gaps and self-fulfilling expectations: alternative implication of paid-for training, Economic Inquiry, 51(3), 1829-1848

Escriche, L. 2007. Persistence of Occupational Segregation: the Role of the Intergenerational Transmission of Preferences. Economic Journal, 520, 837-857.

Escriche, L., Olcina, G. \& Sánchez, R. 2004. Gender discrimination and intergenerational transmission of preferences. Oxford Economic Papers, 56, 485-511.

Fernández, R. 2014. Women's rights and development. Journal of Economic Growth, 19, 37-80.

Fernández, R., Fogli, A., \& Olivetti, C. 2004. Mothers and Sons: Preferences Formation and Female Labor Force Dynamics. Quarterly Journal of Economics, 119, 1249-1299.

Flory, J. A., Leibbrandt, A., \& List, J. A. 2014. Do Competitive Workplaces Deter Female Workers? A Large-Scale Natural Field Experiment on Job-Entry Decisions. The Review of Economic Studies, rdu030.

Galor, O. 2007. Discrete Dynamical Systems. Berlin: Springer.

Gemici, A., \& Wiswall, M. 2014. Evolution of gender differences in post-secondary human capital investments: College majors. International Economic Review, 55, 23-56.

Gilbert, L., Gallessich, J. \& Evans, S. 1983. Sex of faculty role models and students' self-perceptions of competancy. Sex Roles, 9, 597-607.

Gneezy, U., Leonard, K.L., \& List, J.A. 2009. Gender differences in competition: Evidence from a matrilineal and a patriarchal society. Econometrica, 77, 1637-1664.

Goldin, C. 2006. The Quiet Revolution That Transformed Women's Employment, Education, and Family. American Economic Review, Papers and Proceedings, 96, 1-21.

Gong, B. \& Yang, C.-L. 2012. Gender differences in risk attitudes: Field experiments on the matrilineal Mosuo and the patriarchal Yi. Journal of Economic Behavior ES Organization, 96, 1-21.

Gong, B., Yan, H. \& Yang, C.-L. 2014. Gender Differences in the Dictator Experiment: Evidence from the Matrilineal Mosuo and the Patriarchal Yi. Experimental Economics, doi: 10.1007/s10683014-9403-2. 
Komura, M. 2013. Fertility and endogenous gender bargaining power. Journal of Population Economics, 26, 943-961.

Matsuyama, K. 2002. Explaining Diversity: Symmetry-Breaking in Complementarity Games. American Economic Review, 92, 241-246.

Mead, M. 1935. Sex and temperament in three primitive societies. New York: Morrow.

Saez-Marti, M., \& Zilibotti, F. 2008. Preferences as human capital: Rational choice theories of endogenous preferences and socioeconomic changes. Finnish Economic Papers, 21, 81-94.

Shu, X., Zhu, Y. \& Zhang, Z. 2013. Patriarchy, Resources, and Specialization: Marital DecisionMaking Power in Urban China. Journal of Family Issues, 34, 885-917.

Wohlford, K., Lochman, J. \& Barry, T. 2004. The relation between chosen role models and the self-esteem of men and women. Sex Roles, 50, 575-582.

Wolfers, J. 2006. Diagnosing discrimination: Stock returns and CEO gender. Journal of the European Economic Association, 4, 531-541. 